Journal for ImmunoTherapy of Cancer

\title{
Natural and cryptic peptides dominate the immunopeptidome of atypical teratoid rhabdoid tumors
}

\author{
Ana Marcu, ${ }^{1}$ Andreas Schlosser, ${ }^{2}$ Anne Keupp, ${ }^{3}$ Nico Trautwein, ${ }^{1}$ Pascal Johann,,${ }^{4,5}$ \\ Matthias Wölfl, ${ }^{3}$ Johanna Lager, ${ }^{3}$ Camelia Maria Monoranu, ${ }^{6}$ Juliane S Walz, ${ }^{1,7,8,9}$ \\ Lisa M Henkel, ${ }^{3}$ Jürgen Krauß, ${ }^{10}$ Martin Ebinger, ${ }^{11}$ Martin Schuhmann, ${ }^{12}$ \\ Ulrich Wilhelm Thomale, ${ }^{13}$ Torsten Pietsch, ${ }^{14}$ Erdwine Klinker, ${ }^{15}$ Paul G Schlegel, ${ }^{3}$ \\ Florian Oyen, ${ }^{16}$ Yair Reisner, ${ }^{17}$ Hans-Georg Rammensee, ${ }^{1}$ Matthias Eyrich ${ }^{3}$
}

To cite: Marcu A, Schlosser A, Keupp A, et al. Natural and cryptic peptides dominate the immunopeptidome of atypical teratoid rhabdoid tumors. Journal for ImmunoTherapy of Cancer 2021;9:e003404. doi:10.1136/jitc-2021-003404

- Additional supplemental material is published online only. To view, please visit the journal online (http://dx.doi.org/10. 1136/jitc-2021-003404).

Accepted 26 August 2021
Check for updates

(C) Author(s) (or their employer(s)) 2021. Re-use permitted under CC BY-NC. No commercial re-use. See rights and permissions. Published by BMJ.

For numbered affiliations see end of article.

Correspondence to Dr Matthias Eyrich; eyrich_m@ukw.de

\section{ABSTRACT}

Background Atypical teratoid/rhabdoid tumors (AT/RT) are highly aggressive CNS tumors of infancy and early childhood. Hallmark is the surprisingly simple genome with inactivating mutations or deletions in the SMARCB1 gene as the oncogenic driver. Nevertheless, AT/RTs are infiltrated by immune cells and even clonally expanded T cells. However, it is unclear which epitopes T cells might recognize on AT/RT cells.

Methods Here, we report a comprehensive mass spectrometry (MS)-based analysis of naturally presented human leukocyte antigen (HLA) class I and class II ligands on 23 AT/RTs. MS data were validated by matching with a human proteome dataset and exclusion of peptides that are part of the human benignome. Cryptic peptide ligands were identified using Peptide-PRISM.

Results Comparative HLA ligandome analysis of the HLA ligandome revealed 55 class I and 139 class II tumor-exclusive peptides. No peptide originated from the SMARCB1 region. In addition, 61 HLA class I tumor-exclusive peptide sequences derived from non-canonically translated proteins. Combination of peptides from natural and cryptic class I and class II origin gave optimal representation of tumor cell compartments. Substantial overlap existed with the cryptic immunopeptidome of glioblastomas, but no concordance was found with extracranial tumors. More than $80 \%$ of AT/RT exclusive peptides were able to successfully prime $\mathrm{CD}^{+} \mathrm{T}$ cells, whereas naturally occurring memory responses in AT/RT patients could only be detected for class II epitopes. Interestingly, $>50 \%$ of AT/RT exclusive class II ligands were also recognized by $T$ cells from glioblastoma patients but not from healthy donors.

Conclusions These findings highlight that AT/RTs, potentially paradigmatic for other pediatric tumors with a low mutational load, present a variety of highly immunogenic HLA class I and class II peptides from canonical as well as non-canonical protein sources. Inclusion of such cryptic peptides into therapeutic vaccines would enable an optimized mapping of the tumor cell surface, thereby reducing the likelihood of immune evasion.

\section{BACKGROUND}

Atypical teratoid/rhabdoid tumors (AT/ RTs) are malignant tumors of infancy and early childhood. They represent $50 \%$ of brain tumors in children under 1 year. Despite multimodal therapy, consisting of neurosurgery, polychemotherapy and, whenever possible in these small children, radiotherapy, 5-year event-free survival is only $30 \%$. $^{1}$ One hallmark of this cancer are the surprisingly simple genomes, the oncogenic driver is usually restricted to a homozoygous deletional mutation in the SMARCB1 gene. ${ }^{2}$ More recently, AT/RTs have been categorized into the TYR, SHH, and MYG subgroups, which display distinct molecular and clinical features. ${ }^{3} 4$ In cancer mutation rankings, AT/RTs are frequently considered the human cancer with the lowest mutational load. ${ }^{5}$ Although tumor mutational burden has emerged as an important biomarker for response to immunotherapy in certain adult cancer, ${ }^{6}$ this parameter is far from being exclusive. Immunohistological studies have revealed that $\mathrm{AT} / \mathrm{RTs}$ can contain considerable infiltrations of immune cells including T cells. ${ }^{7}$ More recent RNA-seq data suggested that in the SHH- and MYC subgroups of AT/ RTs macrophages play an important role in tumor cell progression ${ }^{8}$ and that these two subgroups also contain gene signatures of clonally expanded $\mathrm{CD} 4^{+}$and $\mathrm{CD}^{+}{ }^{\mathrm{T}}$ cells. ${ }^{9} 10$ Attempts to categorize immune cell infiltrates by DNA methylation data identified three immune microenvironment clusters, but no clear difference between immune cells in AT/RT subtypes. ${ }^{11}$ Finally, tumor-specific $\mathrm{CD}^{+} \mathrm{T}$ cell responses were reported in a small case series of AT/RT patients receiving tumor-specific therapeutic vaccines. ${ }^{12}$ These data provide indications that from an immunological perspective, this tumor entity may not be entirely cold. However, the targets of a possible $\mathrm{T}$ cell recognition are unknown so 
far. To address this issue, we performed a comprehensive analysis of AT/RT specific T cell epitope candidates. Due to the extremely low mutational load in AT/RTs, we considered neoantigens derived from non-synonymous mutations as an unlikely source of candidate peptides and therefore focused our investigation on peptides eluted from human leukocyte antigen (HLA) class I and II molecules of primary tumor tissue. Our intention was to develop a strategy for vaccine design in low mutational tumors in which a maximal representation of the entire ligandome of the tumor cell is given, thereby preventing immune escape. Thus, our mass spectrometry (MS)-based discovery workflow and downstream computational analysis took into account tumor-associated antigens derived from canonical non-mutated or overexpressed natural proteins and antigens from non-canonical or cryptic sequences, which have recently been reported to account for up to $15 \%$ of the immunopeptidome. ${ }^{13}{ }^{14}$ Our data demonstrate that AT/RTs express a plethora of tumorspecific and immunogenic HLA class I and class II ligands from highly diverse origin. Cryptic peptides represent a hitherto untapped source of potential $\mathrm{T}$ cell epitopes that should be considered for personalized tumor vaccine designs.

\section{METHODS}

\section{Patients, tissues and blood samples}

AT/RT tissue samples $(n=18)$ were obtained from tumor banks at the University Hospitals in Würzburg, Bonn, Heidelberg, Berlin, and Tübingen. In five patients treated in Tübingen and Würzburg, fresh tissue could be obtained directly from neurosurgical procedures. According to the requirements of the German HITNetzwerk, AT/RT diagnoses were centrally reviewed and confirmed in all 23 available cases by the DGNN reference neuropathology in Bonn. Tumor tissue samples have been snap frozen and stored in liquid nitrogen until usage. All samples were subject to ligandome analysis as described below. In 10 cases, reference DNA from peripheral mononuclear cells was available; thus, in these cases, whole exome sequencing and neoepitope identification could be performed. In nine tumor samples, MPLA sequencing of the SMARCB1 locus was carried out to identify the nature of the SMARCB1 mutation. The whole workflow of AT/RT tissue workup is detailed in online supplemental figure 1. For immunohistochemistry, 17 additional formalin-fixed and paraffin-embedded AT/RT tumor tissues from the neuropathologies in Würzburg and München were used. Population coverage analysis for HLA class I and II was performed using the IEDB analysis resource (http:// tools.iedb.org/population/). ${ }^{15}$ AT/RT subgroup information was available for 12 of the tissue samples: five belonged to the MYC, four to the TYR, and three to the SHH subgroup, respectively.

\section{HLA typing}

HLA alleles for loci A, B, C, DRB1 and DQB1 were determined by standard sequencing procedures (sequencebased typing SBT, Protrans GmbH, Hockenheim, Germany or GenDx, The Netherlands), which provide high-resolution typing. Confirmatory typing was undertaken by sequence-specific oligonucleotides (SSO, One Lambda or Olrup CareDx, San Francisco, USA) according to institutional regulations.

\section{Immunohistochemistry}

Paraffin-embedded samples (thickness $2 \mu \mathrm{m}$ ) of 17 AT/ RT tissue were deparaffinized with Xylol and Ethanol and subsequently stained with standard immunohistochemistry procedures against CD3 (dilution 1:150), HLA class I (clone EMR 8-5, Abcam, dilution 1:1600), HLA class II (clone CR3/43, Abcam, dilution 1:1000), and PD-L1 (dilution 1:500). One day after primary antibody incubation, slides were stained with streptavidin-peroxidase complex (BioGenex, Fremont, California, USA) for 10 min and counterstained with Mayer's hemalaun solution. Human tissue from tonsil (CD3, HLA class II), liver (HLA class I), and placenta (PD-L1) served as a positive control. Expression levels were evaluated semiquantitatively by two independent investigators and given as the frequency of positive cells per visual field. Ten visual fields per tumor were analyzed. For determining the PD-L1 expression level, the harmonized Cologne scoring system was used. ${ }^{16}$

\section{Isolation of HLA ligands}

HLA class I and clas II molecules were isolated using standard immunoaffinity purification. ${ }^{17}{ }^{18}$ In brief, snapfrozen AT/RT tissue samples were minced in lysis buffer containing $10 \mathrm{mM}$ CHAPS/PBS (AppliChem, St. Louis, Missouri, USA/Gibco, Carlsbad, California, USA) and one protease inhibitor tablet (Complete; Roche, Basel, Switzerland). We employed the pan-HLA class I specific antibody W6/32 ${ }^{19}$ and the HLA-DR specific antibody L243, ${ }^{20}$ both produced in house (University of Tübingen, Department of Immunology) from HB-95, and HB-55 hybridoma cell lines (ATCC, Manassas, Virginia, USA) respectively. Furthermore, the pan-HLA class II antibody Tü39 was employed ${ }^{21}$ and produced in house from a hybridoma clone previously described. Antibodies were covalently linked to to CNBr-activated Sepharose (GE Healthcare, Chalfont St Giles, UK). HLA molecules were purified by cyclically passing the cleared protein lysate from AT/RT tissue over two columns. The first column contained $1 \mathrm{mg}$ of W6/32 antibody coupled to sepharose, whereas the second column contained equal amounts of Tü39 (0.5 mg) and L243 (0.5 mg) antibody coupled to sepharose HLA-DR1 is known to be expressed at higher levels than the other class II allotypes; therefore, the specific L243 mAb at the given stoichiometry was used. ${ }^{22}$ Tü39 is used complementarily to pull down the remaining class II complexes. HLA-peptide complexes were eluted by repeated addition of $0.2 \%$ trifluoroacetic acid (TFA; Merck, Whitehouse Station, New Jersey, USA). Eluted 
HLA ligands were purified by ultrafiltration using centrifugal filter units (Amicon; Millipore, Billerica, Massachusetts, USA) with $3 \mathrm{kDa}$ and $10 \mathrm{kDa}$ pore size for HLA class I and HLA class II ligands, respectively. HLA ligands were desalted using ZipTip C18 pipette tips (Millipore). Extracted peptides were eluted in $35 \mu \mathrm{L} 32.5 \%$ acetonitrile (Merck) $/ 0.2 \%$ TFA, and vacuum-centrifuged to $2-5$ $\mu \mathrm{L}$ and resuspended in $25 \mu \mathrm{L}$ of $1 \%$ acetonitrile $/ 0.05 \%$ TFA. Samples were stored at $-80^{\circ} \mathrm{C}$ until analysis by liquid chromatography-mass spectrometry/mass spectrometry (LC-MS/MS).

\section{Analysis of HLA ligands by LC-MS/MS}

Peptide samples were separated by reversed-phase liquid chromatography (nano-UHPLC, UltiMate 3000 RSLCnano; Thermo Fisher, Waltham, Massachusetts, USA) and analyzed in online-coupled LTQ Orbitrap XL mass spectrometer (Thermo Fisher). Samples were analyzed in three or five technical replicates. Sample shares of $20 \%$ were trapped on a $75 \mu \mathrm{m} \times 2 \mathrm{~cm}$ trapping column (Acclaim PepMap RSLC; Thermo Fisher) at $4 \mu \mathrm{L} / \mathrm{min}$ for $5.75 \mathrm{~min}$. Peptide separation was performed at $50^{\circ} \mathrm{C}$ and a flow rate of $175 \mathrm{~nL} / \mathrm{min}$ on a $50 \mu \mathrm{m} \times 25 \mathrm{~cm}$ separation column (Acclaim PepMap RSLC; Thermo Fisher) applying a gradient ranging from $2.4 \%$ to $32.0 \%$ of acetonitrile over the course of $90 \mathrm{~min}$. Samples were analyzed on an LTQ Orbitrap XL using a top five CID (collisioninduced dissociation) method with survey scans at $60 \mathrm{k}$ resolution and fragment ion detection in the ion trap (ITMS2) operated at normal scan speed. Fragmentation of HLA class I ligands was limited to precursors with a mass range of $400-650 \mathrm{~m} / \mathrm{z}$ and charge states $2+$ and $3+$. For HLA class II ligands, a mass range of $300-1500 \mathrm{~m} / \mathrm{z}$ with charge states $\geq 2$ were selected for fragmentation.

\section{Database search}

The software Proteome Discoverer (V.1.3; Thermo Fisher) was used to perform database search using the Mascot search engine (Mascot 2.2.04; Matrix Science, London, UK). The human proteome as comprised in the Swiss-Prot database (http://www.uniprot.org, status September 2013, 20,225 reviewed sequences contained) was used as reference database. The search combined data of technical replicates and was not restricted by enzymatic specificity. Precursor mass tolerance was set to $5 \mathrm{ppm}$ and fragment mass tolerance to $0.5 \mathrm{Da}$. Oxidation of methionine residues was allowed as a dynamic modification. False discovery rate (FDR) estmation was performed using the Percolator node ${ }^{23}$ and was set to a peptide spectrum match level FDR $\leq 5 \%$. For HLA class I ligands, peptide lengths were limited to 8-12 amino acids (AAs); for HLA class II, peptides were limited to 8-25 AA of length. Protein grouping was disabled, allowing all proteins that contain a given peptide to be annotated as peptide source proteins. Only HLA class I ligands mapping into one source protein alone were considered for this analysis (3244 potential targets). As a final step of quality control, binding prediction was performed employing NetMHC-3.0 with a cut-off score of $\%$ rank $<0.5$ for strong binders and $<2$ for weak binders. ${ }^{24}$

\section{Whole genome DNA sequencing}

Library preparation and whole genome sequencing of tumor and matching normal DNA from peripheral blood was carried out as described previously. ${ }^{25}$ Briefly, $1-5 \mu \mathrm{g}$ of genomic DNA was fragmented to $\sim 300 \mathrm{bp}$ insert size using a Covaris device. Size selection was conducted by agarose gel excision. For paired-end library preparation, we used Illumina, Inc V.2 protocols. Sequencing was performed on HiSeq2000 machines at the German Cancer Research Center (DKFZ) Genomics and Proteomics Core Facility. Sequencing reads were mapped and aligned as describe. ${ }^{3}$ Briefly, we called variants using samtools and vcftools on the tumor bam files. The called positions were looked up in the control bam file, and the resulting raw calls were filtered using different annotations and criteria to create a set of high confidence somatic single nucleotide variants (SNVs). Indels were called using the pipeline platypus. ${ }^{26}$ All coordinates used in the analyses are based on human reference genome assembly hg19, GRCh37 ( ncbi.nlm.nih.gov/assembly/2758). Gene annotations are based on genecode annotation release 19 (gencodegenes. $\mathrm{org} /$ releases/19.html). Whole genome sequencing data have been deposited at the European Genome-phenome Archive, EGA Study Accession IDEGAS00001001297.

The obtained mutated and the corresponding wildtype sequences were verified in Uniprot or Ensemble databases (https://www.uniprot.org/, http://apr2018. archive.ensembl.org/index.html). For each mutation a virtual 9mer-library was created moving the mutated AA from pos 1 to pos 9 and matched with the individual HLA class I type (HLA-ABC) on the NetMHCpan 4.0 server (http://www.cbs.dtu.dk/services/NetMHCpan). For each mutation the best binding peptide was selected according to binding affinity and \% rank.

\section{Immunogenicity prediction tools}

For in silico immunogenicity prediction, two different online platforms were used. VaxiJen2.0 is a publicly available online platform that uses an alignment-independent method for vaccine subunit prediction based on autocross covariance transformation (http://www.ddg-pharmfac. net/vaxijen/VaxiJen/VaxiJen.html). AA properties (hydrophobicity, size and polarity) are transcribed in $\mathrm{z}$ descriptors independently from the corresponding HLA allele. ${ }^{27}$ Protein sequences of source proteins were entered in fasta format, and tumor was selected as target organism. As suggested before, we chose a threshold of 0.45 to define probable protective antigens, slightly higher sensitivity than initially proposed threshold of $0.5{ }^{28}$ In order to consider also HLA restriction, we additionally chose the IEDB immunogenicity prediction tool (http://tools.iedb.org/immunogenicity). ${ }^{29}$ Since the original validation of this algorithm was performed only for nonamer-peptides we restricted our analysis to 9-mers and to the HLA molecules specified in the menu. Peptide 
sequences of potential epitopes were entered, and the $\mathrm{T}$ cell recognition score was determined with a threshold of 0.1 was set to distinguish whether HLA-ligands were predicted to be 'immunogenic' or 'non-immunogenic'. Additionally, the mean $\mathrm{T}$ cell recognition score was calculated for ligandome class I, ligandome cryptic, and neoantigenic peptides in order to compare immunogenicity of these three distinctive groups. Furthermore, the frequencies of probably protective/immunogenic antigens was calculated for the whole group as well as for the subgroups of strong, weak, or non-binders according NetMHC algorithms.

\section{Gene ontology and protein network analysis}

Gene ontology and functional annotations of source proteins were defined and analyzed using DAVID Bioinformatics Resources 6.8, NIAID/NIH (https://david. ncifcrf.gov/summary.jsp). ${ }^{30}$ Protein-protein interaction networks and functional enrichment analysis of proteins from different peptide sources was performed using STRING (https://string-db.org/cgi/input.pl). ${ }^{31}$ For description of protein domains, the PFAM 32.0 database was chosen (https://www.pfam.xfam.org). Overlap of ligandome source proteins was analyzed and visualized using the BioVenn web application (https://www. biovenn.nl). ${ }^{32}$

\section{Naïve $\mathrm{CD8}^{+} \mathrm{T}$ cell priming and $\mathrm{T}$ cell expansion protocol}

For $\mathrm{T}$ cell priming assays. we used a previously described protocol. ${ }^{33}{ }^{34}$ In brief, naïve $\mathrm{CD} 8^{+} \mathrm{T}$ cells were isolated from the peripheral blood of healthy donors positive for HLA-A02*01 $(n=4)$ or HLA-B07*02 $(n=1)$ using a purification of $\mathrm{CD}^{+}$events, followed by depletion with CD45RO and CD57 microbeads on LD and LS columns (Miltenyi, Bergisch Gladbach, Germany). Isolated naïe $\mathrm{CD} 8^{+} \mathrm{T}$ cells were incubated with IL-7 overnight. The next day, T cells were washed and mixed with irradiated, matured autologous dendritic cells (DCs), pulsed with either the heteroclitic HLA-A02*01-restricted peptide Melan-A26-35L as a control or the investigational peptides as indicated at 1 $\mu \mathrm{g} / \mathrm{mL}$. interleukin (IL)-21 (30 ng/mL, Peprotech) was added to the coculture on day 0 the next morning. IL-7 and IL-15 (5 ng/mL, Peprotech) were added on days 3, 5, and 7 , and cells were harvested on day 10 , some of them after restimulation with the original peptide. The MelanA26-35L (ELAGIGILTV) and investigational peptides were purchased from jpt (Berlin, Germany). Harvested $\mathrm{T}$ cells on day 10 or 12 were analyzed for tumor necrosis factor alpha (TNF $\alpha) /$ interferon gamma (IFN $\gamma$ ) positivity. $\mathrm{T}$ cell responses three times above the negative control (autologous DCs without peptide) were considered positive and those above the background but $<3 \times$ were considered intermediate.

$\mathrm{T}$ cell responses defined by specific IFN $\gamma$ secretion were measured using a stimulation-expansion-restimulation protocol $^{35}$ : peripheral blood mononuclear cells (PBMCs) were isolated by Ficoll, stimulated with mature, peptide-loaded autologous DCs at a 4:1 (T:DC) ratio and expanded subsequently with IL-15 (5 ng/mL) for 12-14 days. Medium and IL-15 was refreshed every second or third day. On days 7 and 14, PBMCs were restimulated with the same peptide loaded DCs. Negative control was stimulation with empty DCs (mature, autologous DCs without peptide loading). Six hours after the last restimulation, $\mathrm{T}$ cells were analyzed for IFN $\gamma$ production by intracellular cytokine staining. Only T cell responses three times above the negative control (autologous DCs without peptide) were considered positive. In HLA class I assays, the CEFpool peptide mix (jpt, Hamburg, Germany) was used as a positive control.

\section{Identification of cryptic peptides}

Cryptic HLA-I peptides were identified using PeptidePRISM as described recently. ${ }^{13}$ De novo peptide sequencing was performed with PEAKS X (Bioinformatics Solutions Inc, Canada). ${ }^{36}$ Raw data refinement was performed with the following settings: (1) merge options: no merge; (2) precursor options: corrected; (iii) charge options: no correction; (4) filter options: no filter; (5) process: true; (6) default: true; and (7) associate chimera: yes. De novo sequencing was performed with parent mass error tolerance set to $10 \mathrm{ppm}$. Fragment mass error tolerance was set to $0.15 \mathrm{Da}$, and enzyme was set to none. The following variable modifications have been used: oxidation (M), pyro-Glu from $\mathrm{Q}$ (N-term $\mathrm{Q})$, and carbamidomethylation $(\mathrm{C})$. A maximum of three variable posttranslational modifications were allowed per peptide. Up to 10 de novo sequencing candidates were reported for each identified fragment ion mass spectrum, with their corresponding average local confidence score. Because we applied the chimeric spectra option of PEAKS X, two or more TOP10 candidate lists could be assigned to a single fragment ion spectrum. Two tables ('all de novo candidates' and 'de novo peptides') were exported from PEAKS for further analysis.

All de novo sequence candidates were matched against the six-frame translated human genome (hg38) and the threeframe translated human transcriptome (ENSEMBL 90) using Peptide-PRISM. Results were filtered to $10 \%$ FDR for each category (CDS, UTR5, OffFrame, ncRNA, UTR3, intronic, and intergenic). NetMHCpan 4.0 was used to predict binding affinities for all identified HLA-I peptides for all HLA alleles of the corresponding patient. The allele with the minimal rank reported by NetMHCpan was annotated. As per default, we used a cut-off of $0.5 \%$ rank for strong binders and $2 \%$ rank for weak binders.

Identification of cryptic peptides from the glioblastoma (GBM) data $\operatorname{set}^{37}$ has been performed in the same way using Peptide-PRISM. Comprehensive results have been published recently. ${ }^{13}$

Synthetic peptides were analyzed on an LTQ-Orbitrap Velos Pro (Thermo Scientific) equipped with a PicoView Ion Source (New Objective) and coupled to an EASY-nLC 1000 (Thermo Scientific). Peptides were loaded on capillary columns (PicoFrit, $30 \mathrm{~cm} \times 150 \mu \mathrm{m}$ ID, New Objective) selfpacked with ReproSil-Pur 120 C18-AQ 1.9 m (Dr. Maisch) 
and separated with a 30 min linear gradient from $3 \%$ to $30 \%$ acetonitrile and $0.1 \%$ formic acid and a flow rate of $500 \mathrm{~nL} /$ min. MS scans were acquired in the Orbitrap analyzer with a resolution of 30,000 at m/z 400, MS/MS scans were acquired in the Ion Trap analyzer with normal scan rate using CID fragmentation with $35 \%$ normalized collision energy. A TOP10 data-dependent MS/MS method was used; dynamic exclusion was applied with a repeat count of 1 and an exclusion duration of 120s; singly charged precursors were excluded from selection. Minimum signal threshold for precursor selection was set to 20,000. AGC was used with AGC target with a value of $1 \mathrm{e} 6$ for MS scans and 1e4 for MS/MS scans. Lock mass option was applied for internal calibration in all runs using background ions from protonated decamethylcyclopentasiloxane (m/z 371.10124).

\section{Statistical analyis}

Concordance between different IHC markers was analyzed by Pearson linear regression analysis. To linearize distribution of the percentages of marker positive cells in neuropathological slides, data were logit-transformed and plotted by Pearson linear regression using SPSS V.26. The correlation coefficient $r$ specifies strength and direction of the correlation, the $p$ values indicate whether the correlation coefficient $r$ is significantly different from 0 . Differences between two groups were assessed by non-parametric Mann-Whitney $\mathrm{U}$ test assuming unequal variances. For comparison of multiple groups, the non-parametric Kruskal-Wallis rang-sum test was used. For differences within a group, the two-tailed $t$ test was applied. $\mathrm{P}<0.05$ was considered statistically significant.

\section{RESULTS}

\section{Immunohistochemical landscape of AT/RT tissue}

First, we analyzed an independent cohort of 17 AT/RT tissues for the prevalence of T cells, HLA and PD-L1 molecules. Diagnosis of AT/RT was confirmed by central neuropathological review in all cases. The $1.8 \% \pm 5.1 \%$ of all cells within $\mathrm{AT} / \mathrm{RT}$ tissues represented $\mathrm{CD}^{+} \mathrm{T}$ cells. HLA class I and to a lesser degree also class II molecules were found to be expressed on tumor cells. PD-L1 expression level on tumor cells was $5.1 \% \pm 6.8 \%$ as determined by the Cologne scoring system (figure $1 \mathrm{~A}+\mathrm{B}$ ). Linear regression revealed a weak inverse correlation between HLA class I and CD3 expression (figure 1C) and positive correlation between HLA class I and PD-L1 (figure 1D). From one of the freshly obtained tumor samples, we were able to create a primary rhabdoid cell line. On this cell line, we could confirm constitutive expression of HLA class I and IFN $\gamma$-inducible upregulation of HLA class II and PD-L1 molecules (figure 1E).

\section{LC-MS/MS-based immunopeptidome profiling reveals AT/RT- associated HLA source proteins and HLA class I and II ligands eligible for immunotherapy approaches}

Twenty-three AT/RT tumor samples could be retrieved from five tumor cell banks throughout Germany with a centrally confirmed diagnosis of an AT/RT. The cohort included a total of 51 different HLA class I allotypes,
HLA-A*03:01 $(n=9)$ being the most frequent, followed by HLA-A*02:01 $(n=6)$, HLA-C*04:01 $(n=6)$, and HLA$\mathrm{C} * 07: 01(\mathrm{n}=6$, online supplemental figure 2). Among the world's population, $99.9 \%$ of individuals carry at least one HLA class I allotype that is represented within the here analyzed AT/RT cohort (online supplemental figure 2).$^{1538}$ The comparative cohort of benign tissue donors showed an HLA allotype population coverage of $99.8 \%$ (online supplemental figure 2) and matched $100 \%$ of HLA-A allotypes (13/13), $72 \%$ of HLA-B allotypes $(13 / 18)$, and $92 \%$ of HLA-C allotypes $(11 / 12)$ of the AT/RT cohort.

LC-MS/MS analysis of these 23 AT/RT tumor samples identified 7628 unique HLA class I peptides (range 13-1372, mean 459 per sample) (figure 2A) from 4807 source proteins at a local PSM-level FDR of $5 \%$. The in-house HLA class I benign cohort contained 282 tissues covering among others brain $(n=5)$ and cerebellum ( $n=5)$ (HLA Ligand Atlas Paper Version 2017, extended with ligandomes from disease-free ovary). Based on these two datasets, we performed comparative HLA class I ligandome profiling to identify AT/RT-associated source proteins (figure 2B). We identified 55 HLA class I source proteins exclusively presented on AT/RT samples and from these 56 distinct HLA class I peptide ligands as candidates for downstream immunogenicity analysis (figure 2B, online supplemental table $1)$. The majority of AT/RT-associated source proteins (48/55) were individual to each AT/RT tumor, while only six were shared between two patients, and one was shared between tumor and ascites sample of the same patient.

With respect to HLA class II, 30 distinct class II allotypes were identified in the AT/RT patient cohort, with HLADQB1*03:01 $(n=27)$ being the most frequent, followed by HLA-DRB1*03:01 $(\mathrm{n}=20)$ and HLA-DRB1*15:01 $(\mathrm{n}=20)$. Among the world's population, $99.7 \%$ of individuals carry at least one HLA class II allotype encompassed in the AT/ RT cohort, with an average hit number of 3.71 per individual (online supplemental figure 2).

LC-MS/MS analysis of the AT/RT HLA ligandome revealed 3827 distinct HLA class II peptide sequences (range: 9-698, mean per sample 214) (figure 2A) originating from 1517 source proteins at 5\% local PSM-level FDR. Our HLA class II benign cohort entails 132 ligandomes from benign samples, covering among other tissues, 5 cerebellum and 5 brain samples. Unfortunately, most benign HLA class II ligandomes lack HLA class II typing. By comparing the AT/RT and benign tissue cohort regarding single mapped source proteins, we were able to identify 139 AT/RT-associated HLA class II source proteins associated to 122 distinct HLA class II ligands (figure 2C, online supplemental table 1). Remarkably, multiple AT/RT-associated source proteins were shared among patients: one was presented in 8 different AT/RT samples, 3 additional source proteins were shared between four AT/RT patients and 11 were shared between two patients. As observed for HLA class I AT/RT-associated 
A

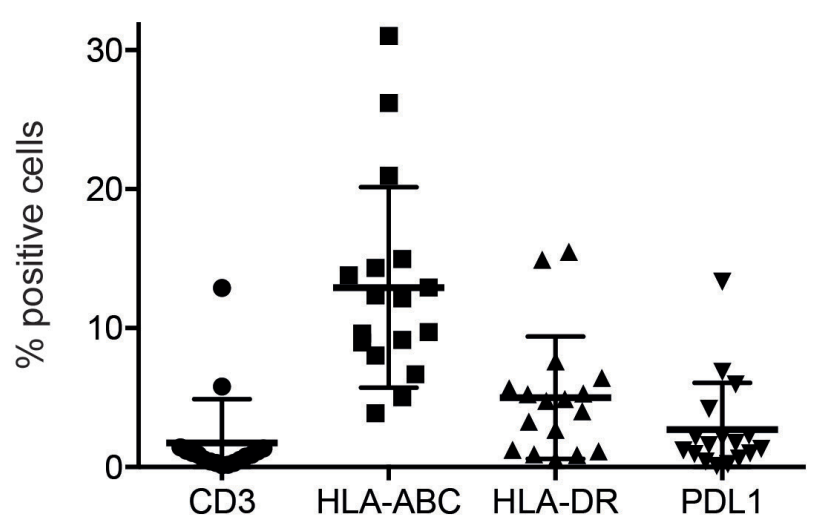

C

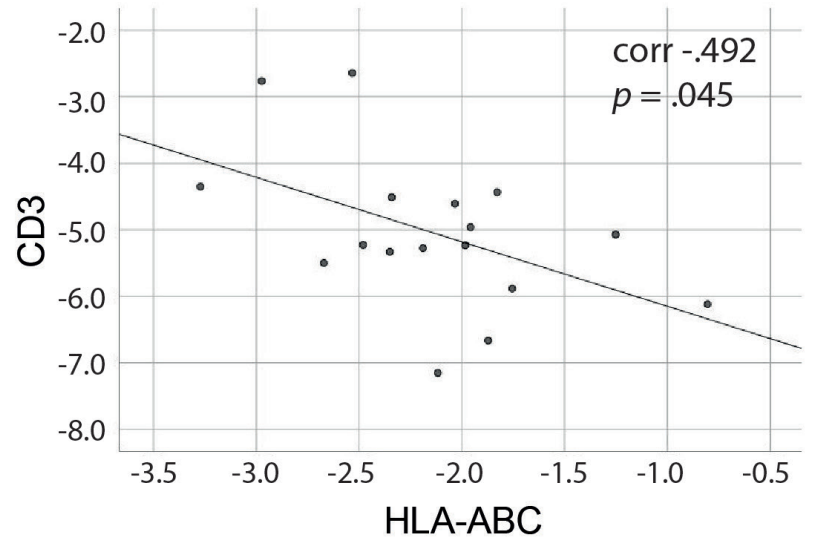

B

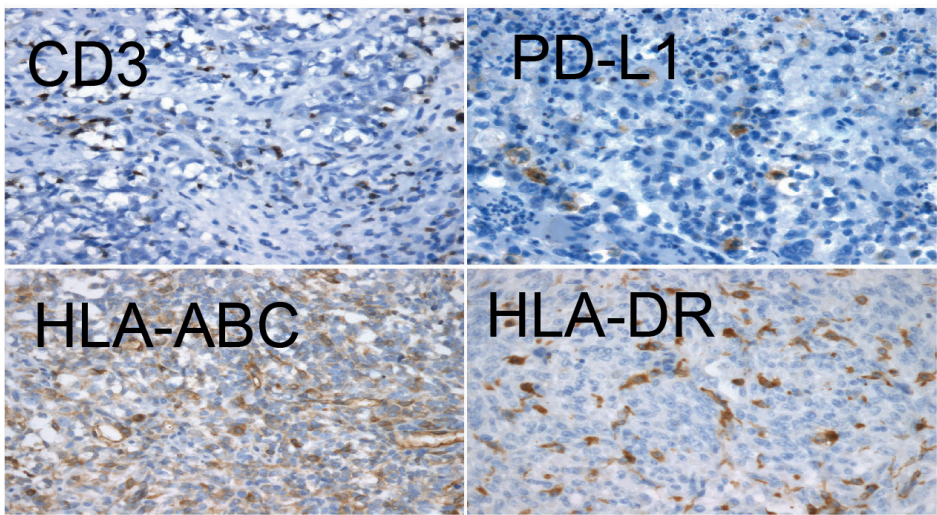

D

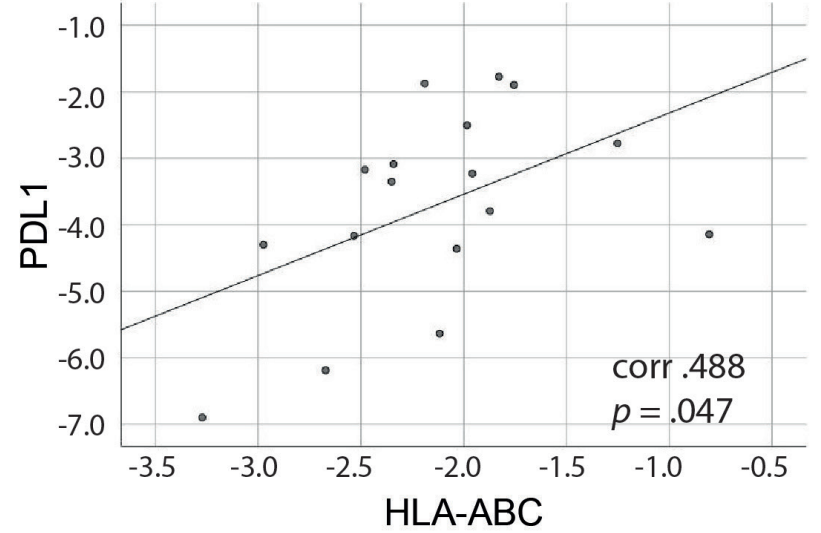

E

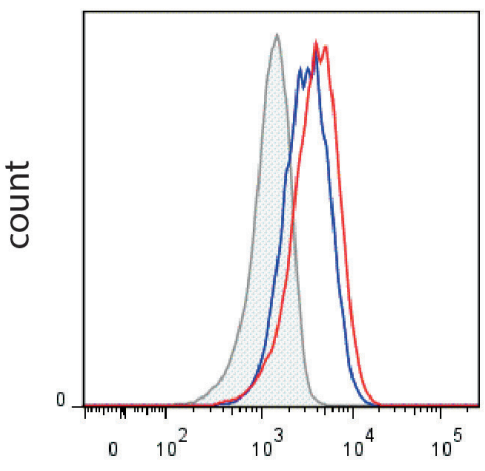

HLA-ABC PE

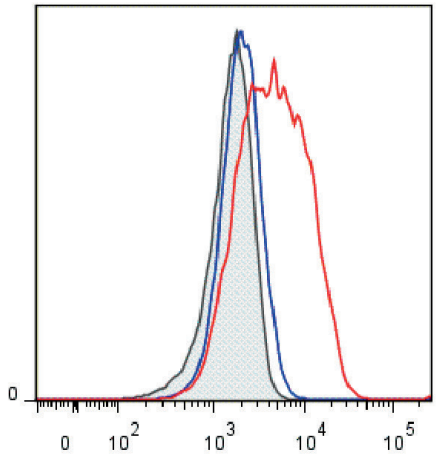

HLA-DR PerCp-Cy5.5

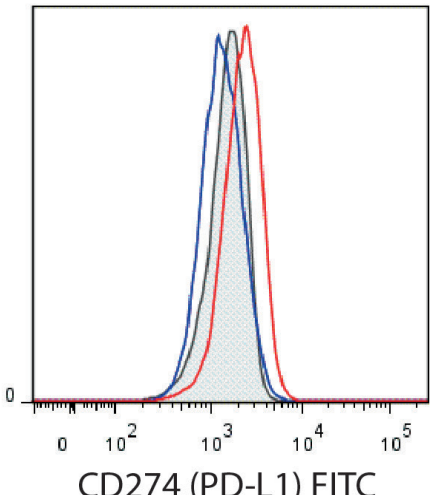

CD274 (PD-L1) FITC

Figure 1 Immunohistochemistry of AT/RT tissues. Seventeen AT/RT tissues were evaluated for expression of CD3, HLA-ABC, HLA-DR, and PD-L1 on tumor cells. (A) Mean values \pm SD for all four markers, 10 visual fields were evaluated in each tumor sample. (B) Representative examples for each of the four markers. (C and D) Linear regression analysis of HLA-ABC expression against $\mathrm{CD}^{+} \mathrm{T}$ cell infiltration (C) and PD-L1 expression (D). Data are plotted after logit-transformation, correlation coefficient $r$ indicates strength and direction of the concordance, the $\mathrm{p}$ value specifies the difference of $r$ from 0 . (E) Flow cytometric analysis of one primary rhabdoid cell line. Shaded line represents unstained control, blue line represents constitutive expression, red line represents expression after IFN $\gamma$ stimulation of tumor cell line. AT/RT, atypical teratoid/rhabdoid tumor. 
A

HLA Class I and II Peptide Identifications
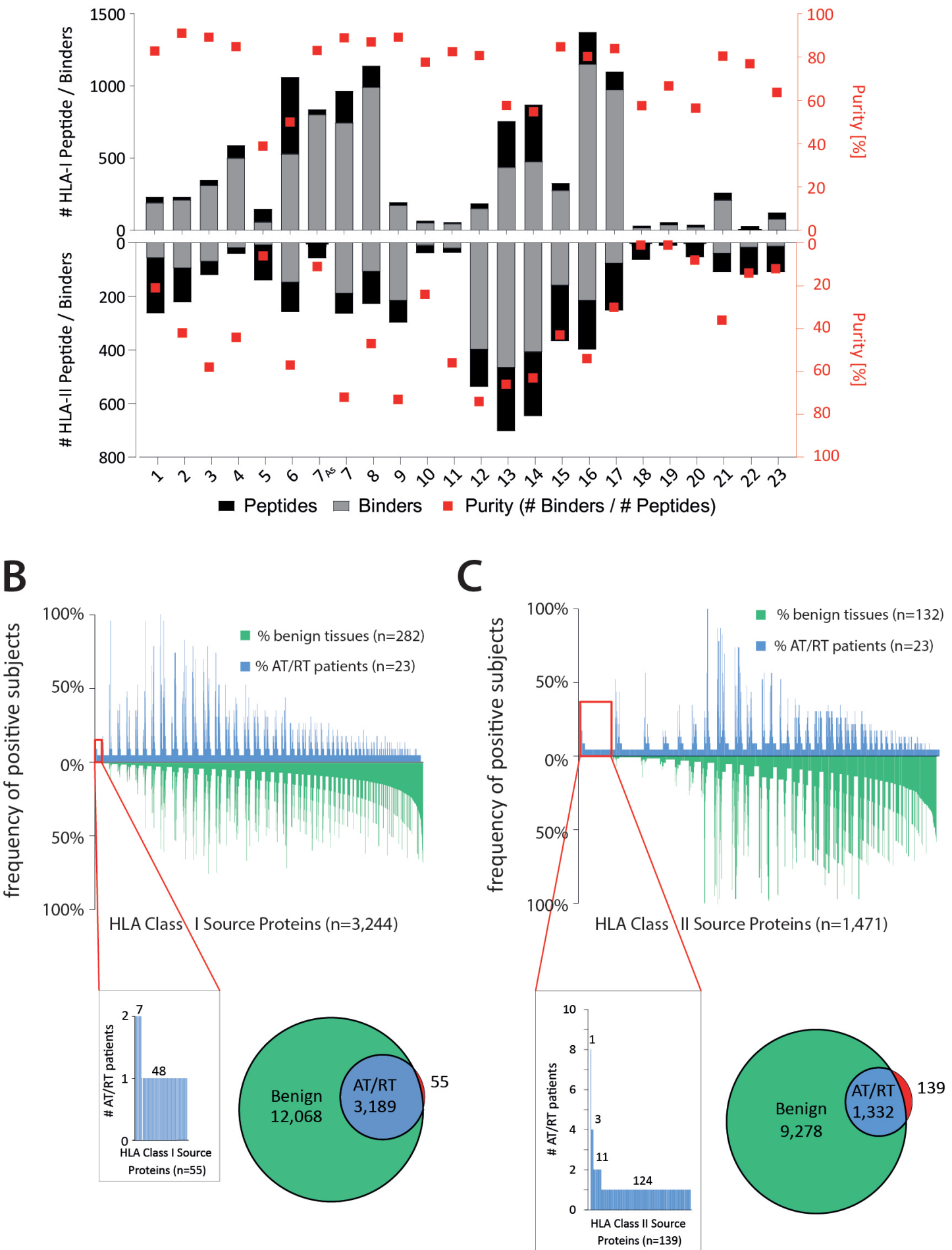

Figure 2 HLA class I and II peptide identifications (A) number of identified peptides in each tumor sample for HLA class I (upward columns) and class II (downward columns). Gray versus black shading illustrates predicted or non-predicted binding of peptides to self-HLA allotypes in the respective patients. Red squares (right y-axis) show purity of peptide pools, that is, the ratio of predicted binding versus total number of identified peptides. (B) Waterfall plot demonstrating overall source protein identifications for HLA class I ligands (x-axis) in benign (green, downward columns) and AT/RT tissue (blue, upward columns) and their frequency in the respective patient cohort. Tumor-exclusive are only the source proteins in the far left red boxes that have no corresponding counterpart in any benign tissue (red parts of Venn diagrams). (C) Same illustration for HLA class II source proteins. 
source proteins, the majority (124) was unique to each patient (figure 2C).

\section{Cryptic peptides in AT/RTs ligandome}

Next, we extended our search to identify cryptic HLA class I peptides from allegedly non-coding regions of the human genome. To this end, we applied a recently introduced approach (Peptide-PRISM) that combines de novo peptide sequencing, highly efficient string search, mixture modeling, and search space stratified filtering of the FDR. ${ }^{13}$ De novo sequencing was performed for all LC-MS/MS data of the 23 AT/RT tumors samples with PEAKS X. Peptide-PRISM was used to match all de novo sequencing candidates against the three-frame translated human transcriptome and the six-frame translated human genome. Two hundred and nine cryptic peptides were identified (FDR 10\%), 158 of which were predicted as binder (rank $<2 \%$ ) to one of the corresponding patient HLA alleles by NetMHCpan 4.0. The distribution of the 158 cryptic peptides among the different categories is shown in figure $3 \mathrm{~A}$. The highest number of peptides derived from non-canonical open reading frames close to the canonical translation start site (categories UTR5 and OffFrame). These cryptic peptides presumably originate from translational misinitiation. An example of such a peptide is shown in figure $3 \mathrm{~B}$.

In order to identify potentially tumor-associated or tumor-specific cryptic peptides, we filtered out all cryptic peptides that have previously been identified within the human HLA ligand atlas project (https://hla-ligandatlas.org) ${ }^{39}$ Sixty-one of the 158 cryptic binding peptides were identified only in AT/RT tumor samples but not in benign tissue (online supplemental figure 2). In addition, we compared the cryptic peptides identified in AT/ RT samples with cryptic peptides previously identified in GBM tumor tissue as well as plasma-soluble HLAs. ${ }^{37} \mathrm{~A}$ complete list of all cryptic peptides from these samples has been recently published. ${ }^{13}$ Twenty of the 61 (33\%) cryptic peptides have been recurrently identified in either at least two AT/RT patients $(9 / 61,15 \%)$ or AT/RT and GBM patients $(11 / 61,18 \%)$, for example, the cryptic peptide LSLEEGIVEV derived from an intron of HIPK3 is identified recurrently in five AT/RT patients (online supplemental figure 2). We selected six cryptic peptides from different categories for analyzing their immunogenicity (see further). Fragment ion spectra of these six synthetically preparated peptides were generated for validating the peptide identification. For all but one peptide (MPISLVQLL) comparison of the fragment ion spectra obtained from AT/RT samples with the fragment ion spectra of the corresponding synthetic peptide confirmed the initial peptide identification (online supplemental figure 3).

Analysis of the distribution of the different peptide classes (class I natural vs cryptic and class II) to the overall ligandome composition between the established A/RT molecular subgroups (MYC, SHH, and TYR) revealed no significant differences compared with the group as a whole (figure 3C) .

\section{Non-synonymous mutations in AT/RTs}

Furthermore, we were interested to examine whether the oncogenic SMARCB1 mutation can give rise to immunogenic peptides. After ligandome analysis, additional tissue was left in 18 cases, in nine of these also PBMCs were available. In the cases with tumor tissue only, MPLA sequencing of the SMARCB1 locus was performed. In these assays, only homozygous or heterozygous deletions but no SNVs of the SMARCB1 locus were detectable, making the existence of neoepitopes in this region unlikely (online supplemental table 3). In the other nine AT/RT cases, we isolated genomic DNA from fresh-frozen AT/ RT tissues and corresponding PBMCs for whole-exome sequencing (WES). As expected, the overall number of non-synonymous mutations was low (median 16, range $1-155)$. Five tumors harbored $\leq 7 \mathrm{SNV}$, whereas four tumors displayed a higher number of SNVs $(99,112,137$, and 155). Two of these belonged to the $\mathrm{SHH}$, one each to the TYR and MYC group, respectively. Also in WES, no non-synonymous mutations in the SMARCB1 locus could be identified. The 24 most frequently mutated genes and their chromosomal location are shown in figure 3D.

To identify potential neoantigenic epitopes from these SNVs, overlapping 9-mer peptide libraries moving the mutation from pos 1 to pos 9 were generated and analyzed for predicted binding to all HLA alleles of the individual patient. The peptide with the best binding score for any of the patients' HLA alleles was selected. A complete list of all in silico predicted neoantigens is provided in online supplemental table 4 . None of these neoantigenic peptide sequences could be verified in the MS data.

\section{Immunogenicity of ligandome peptides}

To assess potential immunogenicity of all identified, AT / RT-associated HLA class I peptides, we first compared in silico predicted major histocompatibility complex (MHC) binding affinity using NetMHCpan 4.0. Whereas binding affinity of neoantigenic and ligandome cryptic peptides was almost identical, ligandome-identified canonical class I peptides displayed a significantly higher binding affinity, suggesting that these peptides have undergone an in vivo selection process in the class I peptide loading complex (figure 4A).

MHC-I binding algorithms mainly rely on binding of certain AAs to anchor residues at P1, P2, and P9 of the MHC-I peptide binding groove. Recently, several in silico immunogenicity prediction tools have used alternative computational methods to assess $\mathrm{T}$ cell receptor recognition of a peptide MHC complex, for example, based on the presence of large, aromatic residues at P4, P5, and P6 of a peptide (IEDB database) or the hydrophobicity, molecular size and polarity of whole protein sequences (VaxiJen server). The IEDB database requires knowledge of the individual peptide sequence, and only a limited set of validated HLA alleles can be analyzed with this tool. 
A

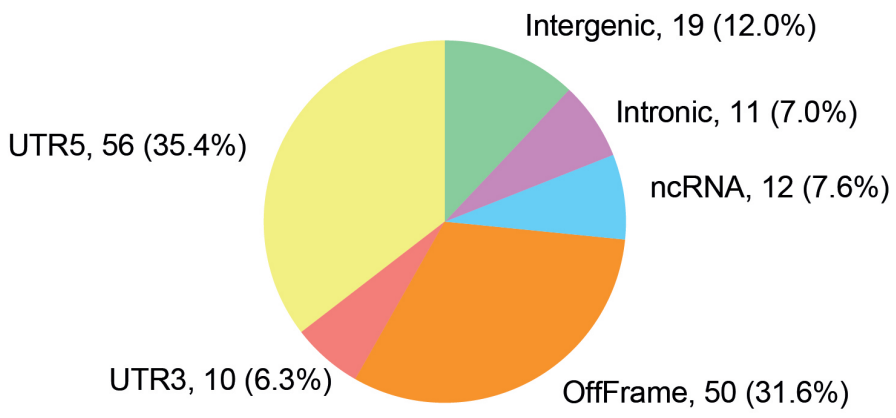

B

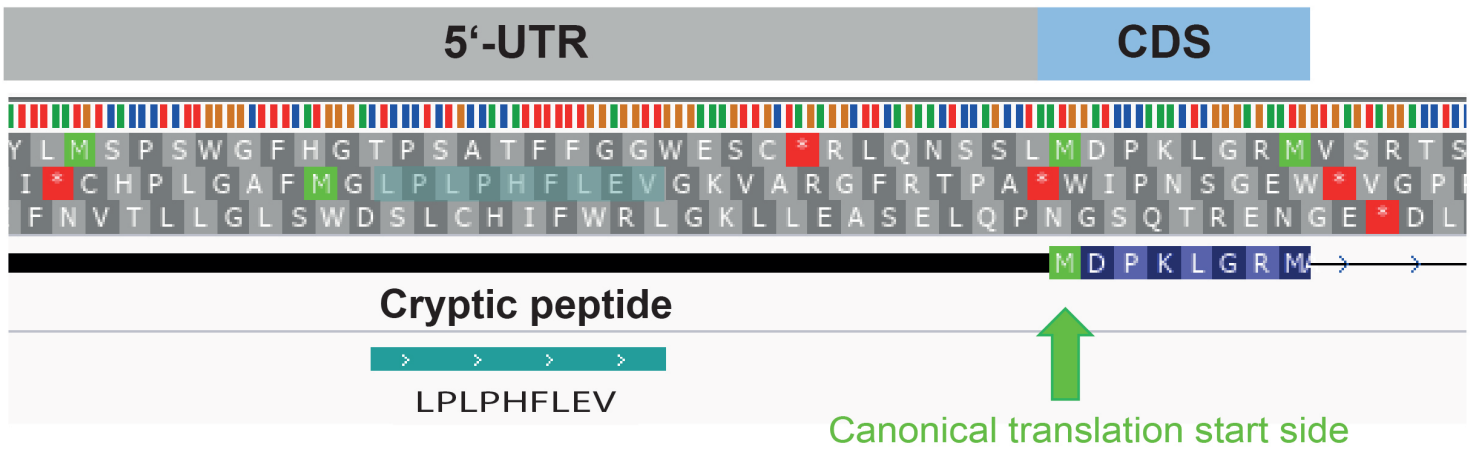

C

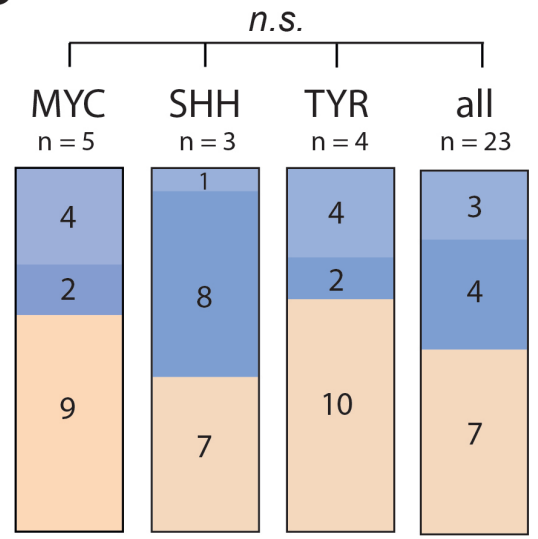

D

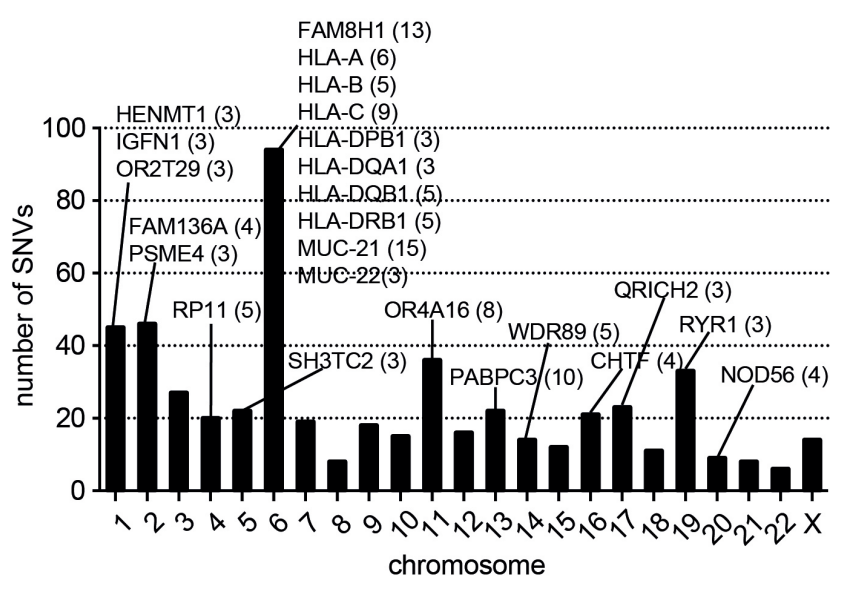

Figure 3 Identification of cryptic class I peptides. One hundred and fifty-eight class I peptides have been identified and predicted as binder to one of the corresponding patient alleles in AT/RT tumor samples. (A) Distribution of identified cryptic peptides among genomic categories. (B) Example of a cryptic HLA peptide from 5'-UTR. The peptide was identified in two AT/ RT patients but not in benign tissue. The peptide is predicted as a strong binder (RANK $<0.5 \%$ ) by NetMHCpan in both patients for HLA-B*51:01 and HLA-B*07:02, respectively. (C) Columns display the relative contribution of different class I and class II peptides to the overall ligandome, numbers indicate the mean number of peptides per group. $P$ values were determined using the non-parametric Kruskal-Wallis test. (D) Most frequently mutated proteins and their localization on the 23 chromosomes. Numbers in parentheses indicate the number of different mutations found in the respective protein. AT/RT, atypical teratoid/ rhabdoid tumor. 
A

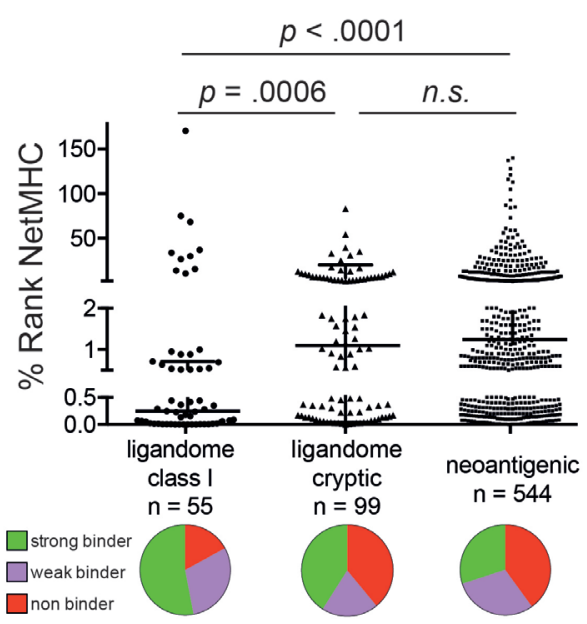

C

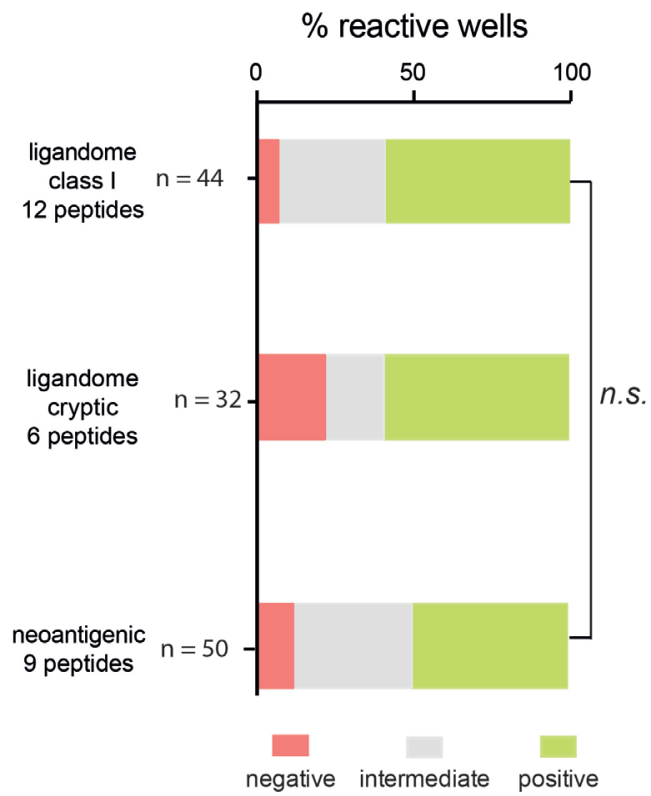

B

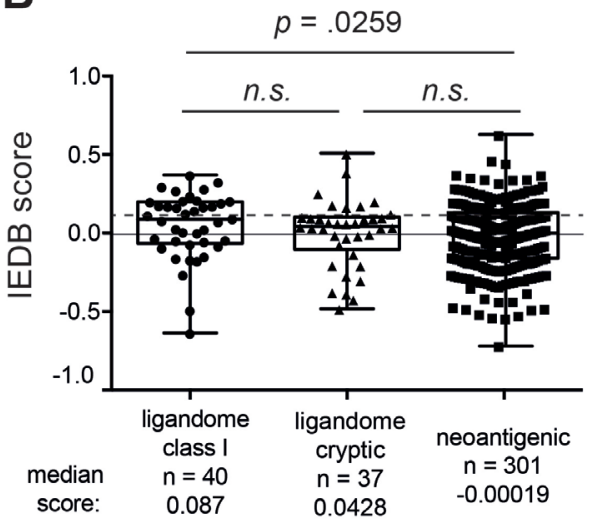

D
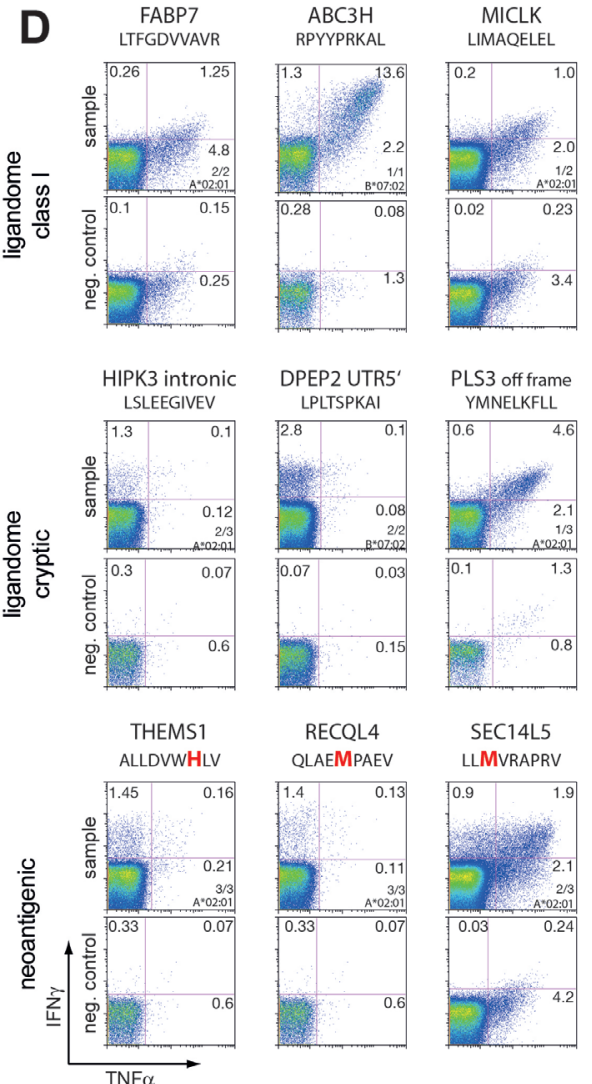

Figure 4 Immunogenicity of HLA class I peptides. (A) Peptides sequences of natural and non-canonical HLA ligands identified by MS as well as in silico predicted neoepitopes were ranked according to the NetMHCpan4.0 algorithm. The best binding HLA allele from each tumor sample was chosen. Pie charts below the graph show classification of \%rank values into strong $(\leq 0.5)$, weak (0.5-2) and non-binders (>2); $n$ indicates the total number of peptides per group. (B) Peptides with an HLA ligand listed in the IEDB data base were alternatively scored for immunogenicity according to the IEDB algorithm; $n$ indicates the number of peptides, which could be submitted to the IEDB server. (C) Highly purified naïve CD8 ${ }^{+} T$ cells from five healthy HLA-A*02:01 $(n=4)$ and HLA-B07*02 $(n=1)$ positive donors were cocultured for 12 days with peptide-loaded autologous DCs in the presence of IL $-7,-15$, and -21 , restimulated and assayed for IFN $\gamma / T N F \alpha$ production. A response $3 \times$ above background (empty dendritic cells (DCs)) was considered as positive (green bars); responses above background but not positive were defined as intermediate (gray bars). $\mathrm{N}$ indicates the total number of wells tested in the respective peptide group. (D) Pseudo-color dot plots display three representative examples of positive responses for ligandome natural, cryptic, and neoantigenic epitopes, respectively. Negative controls (cells stimulated with empty DCs) are shown below each plot. For the complete data set of priming assays, please refer to online supplemental figure 4. Upper numbers in the quadrants show percentages of $C D 8^{+} I F N \gamma^{+}$and $/$or TNF $^{+}$, while specifications in the lower right quadrant indicate number of positive experiments against the amount of donors tested as well as the HLA restriction used. The bold and red colored amino acid indicates the mutation. P values were determined using Mann-Whitney or Kruskal-Wallis test. IFN $\gamma$, interferon gamma; TNF $\alpha$, tumor necrosis factor alpha. 
For IEDB, we considered only peptides with the validated length of nine AAs. IEBD generally yielded lower frequencies of peptides with a positive prediction $(37.8 \%-52.3 \%)$ than the VaxiJen platform $(62.3 \%-77.9 \%)$. Using the IEDB prediction algorithm AT/RT ligandome class I peptides had higher immunogenicity prediction scores than neoantigenic peptide sequences (figure 4B). In contrast, the Vaxijen server favored neoantigenic and ligandome cryptic over ligandome class I source proteins (data not shown). There was no correlation between MHC binding affinity and either IEBD or VaxiJen scores (data not shown).

In addition to these in silico predictions, we tested the immunogenicity of the identified peptides in an in vitro priming assay. This assay uses highly purified naïve $\mathrm{CD} 8^{+}$ $\mathrm{T}$ cells from healthy donors as starting population and autologous DCs as stimulators, thereby closely mimicking a vaccination scenario in vivo. ${ }^{334}$ We focused on two HLA alleles with a high prevalence in our general population (HLA-A*02:01 and HLA-B*07:02) and tested all identified peptides for binding to these two alleles. From the resulting binders we choose 12, 6 , and 9 peptides from the list of ligandome-identified canonical class I, cryptic, and neoantigenic peptide pools, respectively, with a preference for strong binders (23 strong and 4 weak-binders). Ninety-two per cent (11 out of 12), 83\% (5 out of 6 ), and $89 \%$ ( 8 out of 9 ) of the tested peptides yielded at least one positive well with no significant difference between the groups (figure 4C, the complete data set of priming assays is shown in online supplemental figure 4), confirming that the majority of peptides from the different pools can be recognized by naive $\mathrm{T}$ cells. We found a trend for a correlation between predicted HLA binding and cytokine reactivity as well as more cytokine ${ }^{\text {pos }} \mathrm{T}$ cells in strong versus weak binders, but due to the large variation among the strong binders and the low number of selected weak binding peptides, these tests failed to reach significance (online supplemental figure 4).

Furthermore, we investigated whether memory responses against these peptides can be detected in AT/ RT patients. PBMCs from four AT/RT patients were assayed in restimulation experiments for IFN $\gamma$ response against peptides fitting their HLA restrictions. For HLA class I peptides, we screened all peptides for potential binding to HLA-A and HLA-B alleles from these four patients and identified 17, 18, 23, and 32 suitable peptides for restimulation experiments. As demonstrated in figure 5A, only one ligandome peptide (FPILSTILL from the CCG4 source protein) showed a positive response in one patient, whereas all other experiments remained negative, confirming that constitutive $\mathrm{CD} 8^{+} \mathrm{T}$ cell responses against tumor-associated peptides without prior vaccination are exceedingly rare. The same assay was repeated for the 18 HLA class II peptides that were identified more than once in AT/RT tumor samples with gating on $\mathrm{CD} 3{ }^{+} \mathrm{CD} 4^{+} \mathrm{T}$ cells (figure $5 \mathrm{~B}$ ). Notably, 15/18 class II peptides showed at least one positive response, two peptides even gave positive results in three out of four patients tested. To analyze whether these responses are specific for patients with AT/RT, we repeated this experiment with PBMCs from three high-grade glioma (HGG) patients and three healthy controls. In HGG patients, $10 / 18$ peptides yielded a positive response, however, only in one patient each. The LSIEEDVLAA peptide derived from THMS1 source protein (detectable in eight different AT/RT samples) showed a more substantial response in one HGG patient (figure 5B). In PBMCs from healthy controls only eight faint responses at the detection limit in singular patients were measurable. These data indicate that $\mathrm{T}_{\text {helper }}$ cells of AT/RT patients have been exposed to HLA class II ligandome peptides before, which has resulted in memory formation in more than $80 \%$ of tested peptides. This phenomenon seems to be tumor specific as such responses were less detectable in HGG patients and virtually absent in healthy donors. For HLA class I peptides no such memory formation could be observed.

\section{Gene ontology and protein network analysis}

Having identified tumor-exclusive peptides from four different sources (ligandome HLA class I and II peptides from natural proteins, ligandome class I cryptic, and neoantigenic peptides), we mined these data for existing overlap between the respective source proteins or pathway enrichment. Functional annotation using the DAVID Bioinformatics database revealed little similarity in terms of biological process or molecular function (online supplemental table 5). Of note, the four different peptide sources covered distinct cellular compartments. In line with previous reports, HLA class I ligandome peptides were mainly derived from cytosolic proteins, whereas HLA class II ligandome source proteins were allocated to the plasma membrane. AT/RT neoantigens covered external and internal membrane proteins (plasma, Golgi, endo-/lysosomal membranes, junctions, MHC class II complex), whereas source proteins of ligandome cryptic peptides were primarily assigned to the nucleoplasm (figure 6A). Functional enrichment analysis of protein networks showed four, three, and one enriched protein domains within neoantigenic, cryptic and HLA class I source proteins, respectively, whereas for HLA class II no such enrichment could be detected (figure 6B). Likewise, only the network of mutated proteins displayed a significantly higher number of interactions than expected, with a cluster in HLA and HLA-associated genes (figure 6C), which is consistent with the high number of mutations on chromosome six (figure 3C). Taken together, these data indicate that source proteins from different AT/RT-specific peptide pools cover a wide variety of biological functions and show little enrichment of particular protein domains. However, at least in AT/RTs, the different peptide sources seem to emerge from distinct cellular compartments.

\section{Overlap of AT/RT ligandome with other tumor-specific ligandomes}

Finally, we matched our list of HLA class I AT/RT ligandome peptides from natural source proteins with 
A
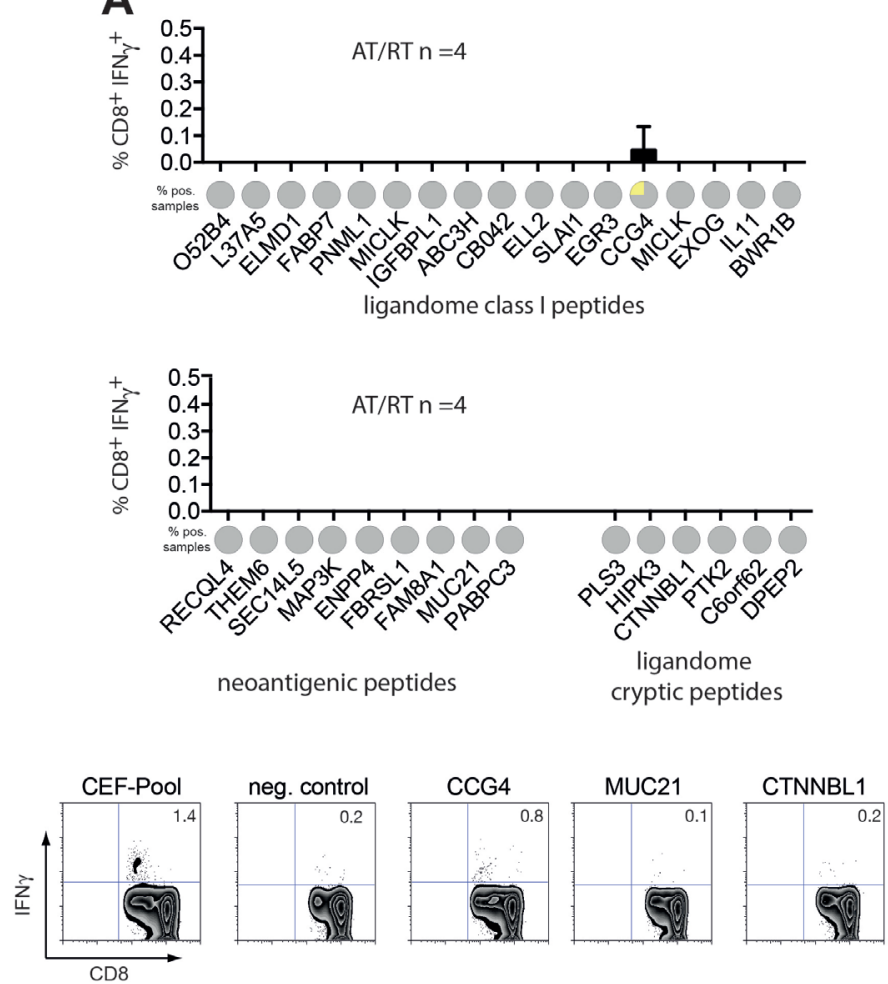

Figure 5 Recall responses against HLA class I and class II peptides in AT/RT patients. (A) Peptides sequences of natural and non-canonical HLA class I ligands identified by MS as well as in silico predicted neoepitopes were screened for binding to the HLA-A and HLA-B alleles of four AT/RT patients, in whom PBMCs were available. The resulting 17, 18, 23, and 32 peptides were used to activate PBMCs from these four patients. Column graphs show frequencies of CD8 ${ }^{+}$IFN $\gamma^{+} \mathrm{T}$ cells after $1-2$ rounds of restimulation. Pie charts above source protein names display the frequency of positive tests ( $>3 \times$ above background, in yellow). Zebra plots below the charts show one representative example of the onliest positive result in a natural ligandome peptide and two negative wells from a cryptic and neoantigenic peptide from the same patient plus controls. (B) The 18 ligandome class II peptides that were detected in more than one tumor sample were used to activate CD4 ${ }^{+} \mathrm{T}$ cells from four AT/RT patients. PBMCs of three patients with high-grade gliomas and three healthy individuals served as controls. Column graphs show frequencies of $\mathrm{CD}^{+} \mathrm{IFN} \gamma^{+} \mathrm{T}$ cells after one to two rounds of restimulation. Pie charts above source protein names display the frequency of positive tests ( $>3 \times$ above background, in yellow). Zebra plots below the charts show five representative examples of positive responses to class II peptides from different AT/RT patients. AT/RT, atypical teratoid/rhabdoid tumor; MS, mass spectrometry; PBMCs, peripheral blood mononuclear cells.

published data sets of tumor-specific ligandomes from other cancer entities. Selection criterion for these comparators was their tumor specificity, that is, the exclusion of peptides also found in other benign tissues. We used two published data sets from GBM, ${ }^{3740}$ and one from ovarian cancer ${ }^{41}$ and renal cell carcinoma ${ }^{28}$ each. While $6 \%$ of source proteins overlapped between the two GBM datasets, only $2 \%$ could be found simultaneously in AT/ RT and GBM. No overlap existed between AT/RTs and the two non-CNS cancers (ovarian and renal; figure 6D). The more substantial overlap $(33 \%)$ between AT/RT and GBM in the cryptic ligandome has been mentioned previously.

\section{DISCUSSION}

Tumor immunosurveillance represents a difficult task for immunity, since cancer cells are a derivate of self and thymic selection has trained $\mathrm{T}$ cells for self-tolerance. The vast majority of the several hundred thousand MHC molecules on a cancer cell is loaded with self-peptides. For tumor eradication, both the affinity between peptide and $\mathrm{MHC}^{42}$ and between TCR and peptide-MHC comple ${ }^{43}$ has been demonstrated to be of critical importance. Therefore, the search for optimal anticancer $\mathrm{T}$ cell epitopes focuses either on high affinity peptides from natural proteins, which are normally not expressed on benign tissue or on peptides derived from sequences, which are not part of the thymic Aire ${ }^{+}$medullary epithelial cell library effective during $\mathrm{T}$ cell selection. From the latter category, peptides covering a cancer-specific mutation have been intensely investigated in recent years. Although $\mathrm{T}$ cell responses against such neoantigens derived from non-synonymous mutations undoubtedly exist and can be boosted by vaccination approaches, ${ }^{44}$ detection of neoantigenic peptides within the HLA ligandome has been exceedingly rare. ${ }^{45}$ One study estimated that less than $1 \%$ of all non-silent mutations are represented in the HLA ligandome. ${ }^{46}$ Given the fact that 
A ligandome MHC class I

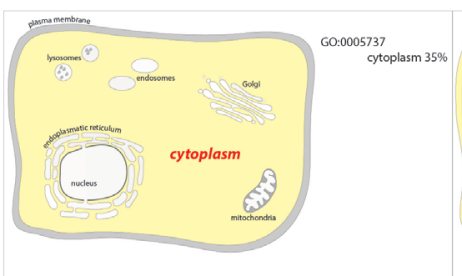

B

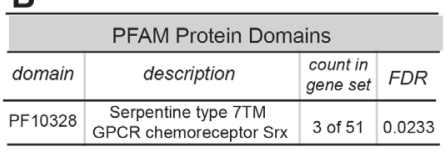

ligandome MHC class II ligandome cryptic peptides neoantigens
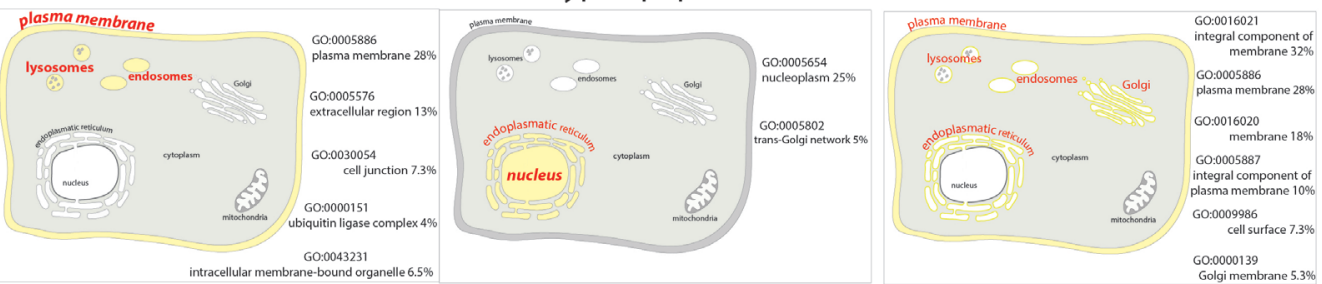

\begin{tabular}{c|c|c|c}
\hline \multicolumn{4}{|c}{ PFAM Protein Domains } \\
\hline domain & description & $\begin{array}{l}\text { count in } \\
\text { gene set }\end{array}$ & FDR \\
\hline- & none detected & - & - \\
\hline
\end{tabular}

\begin{tabular}{l|c|c|c}
\hline \multicolumn{4}{|c}{ PFAM Protein Domains } \\
\hline domain & description & $\begin{array}{c}\text { count in } \\
\text { gene set }\end{array}$ & $F D R$ \\
\hline PF00069 & Protein kinase domain & 8 of 481 & 0.0194 \\
\hline PF02469 & Fasciclin domain & 2 of 4 & 0.0194 \\
\hline PF07714 & Protein tyrosine kinase & 8 of 482 & 0.0194 \\
\hline
\end{tabular}

\begin{tabular}{c|c|c|c}
\hline \multicolumn{4}{c}{ PFAM Protein Domains } \\
\hline domain & description & $\begin{array}{c}\text { count in } \\
\text { gene set }\end{array}$ & FDR \\
\hline \begin{tabular}{c|c} 
Immunoglobulin \\
C1-set domain
\end{tabular} & 12 of 45 & $9.50-08$ \\
\hline $\begin{array}{c}\text { PF009654 } \\
\text { Class II histocompatibility } \\
\text { antigen, beta domain }\end{array}$ & 5 of 9 & 0.0006 \\
\hline $\begin{array}{l}\text { PF00094 von Willebrand factor type D } \\
\text { domain }\end{array}$ & 4 of 14 & 0.0268 \\
\hline PF06623 & MHC_I C-terminus & 3 of 4 & 0.0268
\end{tabular}

C
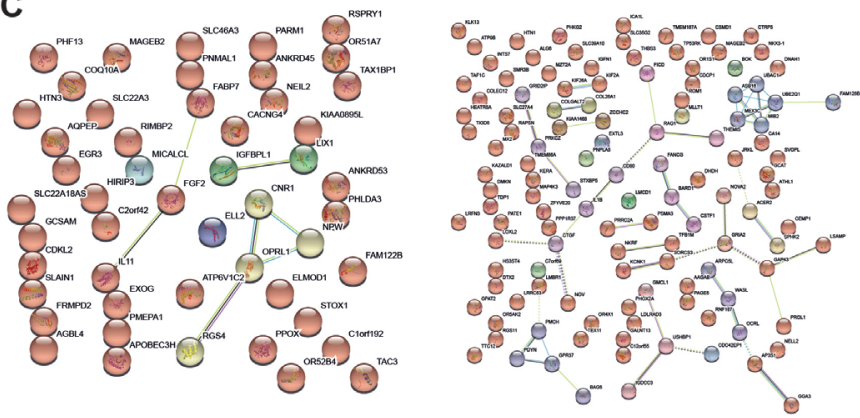

number of nodes: 123

number of edges: 47
number of expected edges: 39
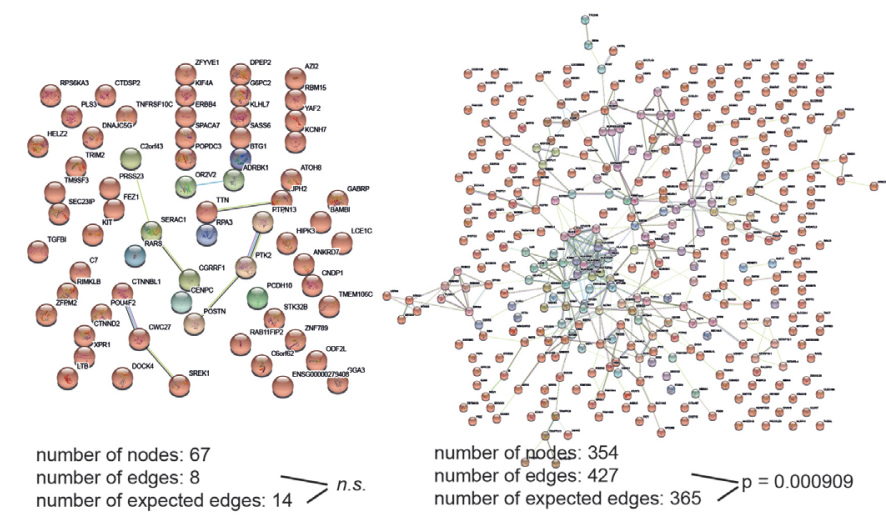

number of nodes: 50

number of edges: 7
number of expected edges: 3 n.s.

number of nodes: 67

number of edges: 8
number of expected edges: 14
n.s.
D

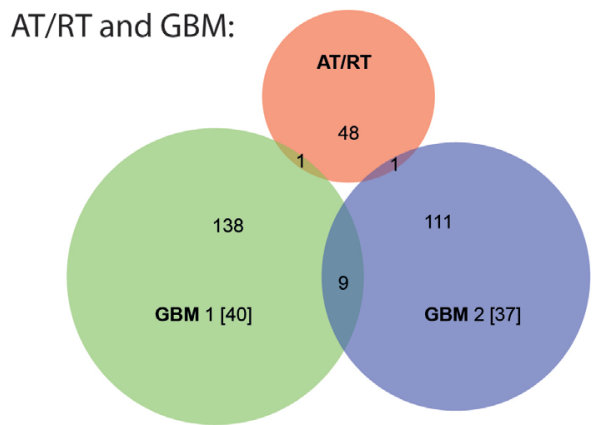

Overlapping source proteins:

$\begin{array}{cc}\text { AT/RT \& GBM 1: } & \text { GBA 1 \& GBM 2: } \\ \text { FABP7 } & \text { ADAM17 } \\ & \text { APC } \\ \text { AT/RT \& GBM 2: } & \text { CCNB1 } \\ \text { ANKRD45 } & \text { CSPG4 } \\ & \text { EGFR } \\ & \text { IGF2BP3 } \\ & \text { ITGB8 } \\ & \text { SPAG9 } \\ & \text { TOP2A }\end{array}$

AT/RT and non-CNS tumors:

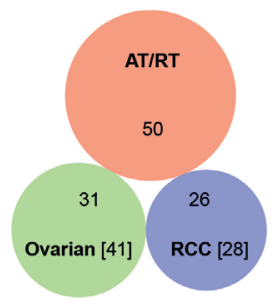

Figure 6 Gene ontology and network analysis of AT/RT peptides. (A) Functional annotation 'cellular compartment' of the four different AT/RT peptides sources defined by DAVID Bioinformatics Resources 6.8. (B) Listing of enriched protein domains in the four source protein cohorts. (C) Protein network analysis using string. (D) Overlap of the AT/RT peptide pool with four published cancer immunopeptidome data sets: GBM $1,{ }^{40} \mathrm{GBM} 2,{ }^{37}$ ovarian cancer ${ }^{41}$ and renal cell carcinoma. ${ }^{28}$ AT/RT, atypical teratoid/ rhabdoid tumor.

most tumors harbor less than 100 somatic mutations and that at least three identical neighboring peptide-MHC complexes are required to trigger cytotoxicity, ${ }^{47}$ the search for neoepitopes has been extended to so-called non-mutated neoantigens, that is, epitopes derived from non-canonical translated or novel protein isoforms. ${ }^{48}$ This new category of HLA-bound epitopes has originally been discovered in the ligandome of normal cells but recently also confirmed in adult tumors. ${ }^{49} 50$ Pediatric cancers generally rank among tumors with a low mutational burden. AT/RTs are a typical representative of this category, as one deleting mutation in the SMARCB1 gene is sufficient for oncogenesis. ${ }^{51}$ Since SMARCB1 deletion leads to widespread epigenetic dysregulation, AT/ RTs are considered epigenetic diseases. Yet, AT/RTs are infiltrated by immune cells, ${ }^{7}$ and subgroups of AT/RTs, especially the MYC and SHH subsets, ${ }^{911}$ even show a preference for cytotoxic T cells. ${ }^{9}{ }^{10}$ Our IHC data confirm that approximately $2 \%$ of all cells within the AT/RT microenvironment are $\mathrm{T}$ cells. The negative correlation 
between CD3 and HLA-ABC could be interpreted as HLA-downmodulation as part of a reversible, epigenetically regulated tumor escape mechanism. ${ }^{52}$ However, the accumulation of mutations on chromosome 6 and the identification of MHC mutations as the only significant network cluster in gene ontology analysis of WES data could also point toward partial HLA loss as an AT/RT-intrinsic 'hard' lesion. ${ }^{53}$ This issue, including strategies to restore HLA expression, warrants further investigation. Furthermore, our data confirm that neoepitopes derived from non-synonymous mutations are not presented on the surface of AT/RT cells (at least not within our range of detection), as no SMARCB1-derived peptide could be identified. All analyzed patients in our cohort exhibited truncating mutations, making generation of mutationderived peptides even more unlikely. Unexpectedly, four AT/RTs showed 100 or more mutations with no preference for one of the recently consented MYC, SHH, and TYR subgroups, ${ }^{3}{ }^{4}$ but also in these cases, no neoantigenic peptide could be confirmed in the ligandome data. Furthermore, we could not find differences in peptide composition between the subgroups, confirming DNA methylation data that rather the type of immune cell infiltration than the subgroup determines survival outcome. ${ }^{11}$ How the ligandome shapes different immune cell infiltration patterns remains to be elaborated in future studies.

Ligandome analyses have been performed in several adult cancer types such as melanoma, ${ }^{45} \mathrm{GBM}^{40}$ colorectal, ${ }^{54}$ lung, ${ }^{55}$ renal cell cancer (RCC), ${ }^{56}$ or ovarian cancer ${ }^{41}$ as well as in hematological malignancies such as multiple myeloma ${ }^{57}$ and leukemias. ${ }^{58}$ Our study in AT/ RTs represents the first pediatric tumor entity analyzed. As in their adult counterparts, the AT/RT ligandome is primarily composed of peptide ligands from a wide variety of natural proteins with little overlap between individual samples, making it unlikely that entity-spanning universal HLA class I epitopes do exist. Overlap was higher for HLA class II peptides, as epitopes derived from THEMIS, AAGAB, ASB16, and EXTL3 proteins were found in more than two tumors in our cohort. These targets can be regarded as bona fide AT/RT HLA-II ligands, since identification by MS has demonstrated highest fidelity for ligands with real MHC II binding. ${ }^{59}$ For HLA-I, only four peptides were detected in two tumors; all remaining were unique to one specific tumor. A small overlap existed with two published GBM data sets, suggesting that the ligandome of CNS tumors is more closely related than that of extracranial cancers. No viral, or retroviral sequences as suggested, ${ }^{10}$ could be identified in the AT/RT immunopeptidome. With regard to vaccine development, these data highlight the necessity to characterize the individual HLA ligandome of each tumor as part of a personalized molecular diagnostic workup. Given the higher overlap within the HLA class II peptidome and the broader reactivity of $\mathrm{CD}^{+} \mathrm{T}$ cells, it is conceivable that an HLA class II peptide mix might cover the majority of AT/RT patients.
However, such an approach requires more ligandome and immunogenicity data.

Our epitope discovery platform additionally included the search for peptides derived from non-canonical protein translation. Such false or aberrant translation events result in small and extremely short-lived intracellular proteins, which are hard to detect by conventional proteomics but can nevertheless enter the proteasomal pathway. Intriguingly, half-life of a cryptic pMHC complex is about 90 times longer than that of the cytosolic source protein. ${ }^{60}$ Cryptic peptides have recently been reported to make up to $15 \%$ of the HLA class-I ligandome in normal as well as in malignant cells. ${ }^{13} 14$ HLA class II cryptic peptides are unlikely to exist since class II epitopes are longer and derived from large and stable internalized extracellular proteins. ${ }^{61}$ Cryptic peptides might represent a new and preferential source of targetable antigens for cancer immunotherapy, ${ }^{49}$ as they are abundant, tumor specific and possibly shared between different tumors. ${ }^{61}$ Since they are most likely not part of the library of selfpeptides presented during thymic $\mathrm{T}$ cell selection, they can be regarded as non-mutated neoantigens. We were able to identify 61 tumor-specific, HLA-binding cryptic peptides not being present in the benign dataset, ${ }^{39}$ thus, more than half of the 116 HLA class I peptides in AT/RTs originated from non-canonical source proteins. Out-offrame translations and gene products from $5^{\prime} \mathrm{UTR}$ regions dominated as reported previously. ${ }^{14}$ HLA peptides categorized as 'intronic' are not necessarily derived from intron retentions but might also derive from an overlapping aberrant transcript that is located just by chance within an intronic region. Furthermore, since our approach did not include RNA-seq analysis, we could not identify neojunction peptides derived from alternative splicing sites, since the latter are not part of the ENSEMBLE 90 database. Future studies will have to include RNA-seq data to allow identification of this emerging class of novel tumor antigens. Thirty-three per cent of these peptides were repeatedly found either in several AT/RT samples or in a published GBM dataset. ${ }^{13}$ Notably, the peptide repertoire overlap between AT/RT and GBM was higher for cryptic than for natural HLA ligands. HLA-I binding prediction showed that ligandome peptides from natural proteins had significantly stronger HLA binding than cryptic peptides, indicating that the former have undergone a more stringent intracellular selection process. Noteworthy, when we combined peptide ligands from all three subtypes, suitable ligands were found for all patients with a median of 13 peptides per tumor (range 3-34), indicating that even low-mutational pediatric tumors give rise to actionable tumor-associated $\mathrm{T}$ cell targets.

In contrast to other studies, which detected small sets of entity-spanning antigens in hematological malignancies $^{58}$ and GBMs, ${ }^{62}$ we could not identify network clusters for HLA I or II ligands using gene ontology tools. Since these algorithms were trained on larger data sets, it is possible that we missed them due to the rather low number of source proteins in our data $(<140)$. However, 


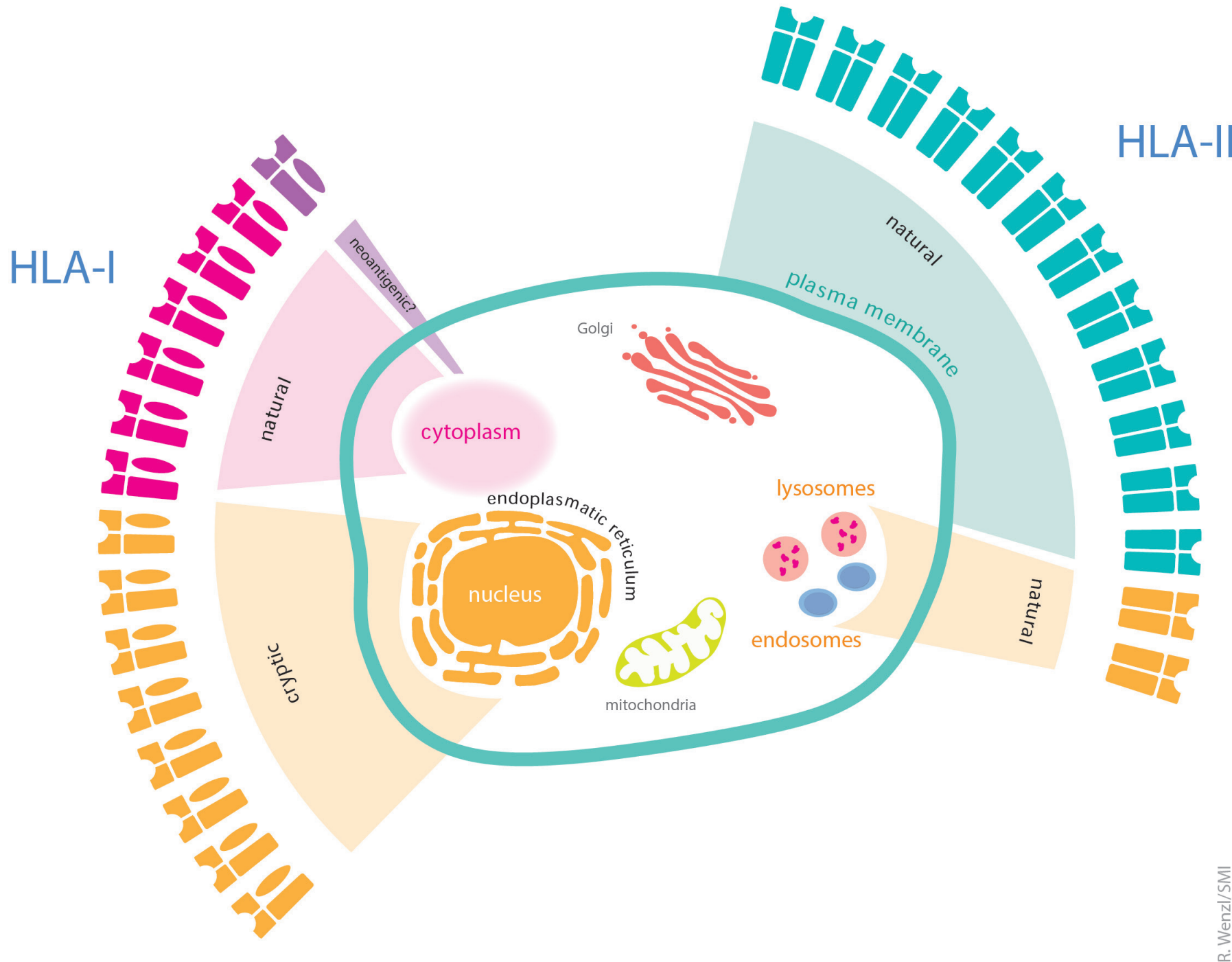

Figure 7 The immunological homunculus of AT/RTs. Illustration of the immunological representation of different cellular compartments of the AT/RT cell on HLA class I (left) and HLA class II molecules (right). Portion of the respective wings reflects the contribution of each peptide source to the class I or II peptidome. The size of the compartments in the outer circle corresponds to the representation of the compartment in the peptidome. AT/RTs, atypical teratoid/rhabdoid tumor.

the allocation of source proteins to specific cellular compartments followed clear patterns. As expected, source proteins for natural class I ligands were mainly located in the cytoplasm, whereas class II peptides derived from membrane bound or intracellular vesicle associated proteins. Cryptic peptides were annotated to the nucleoplasm and to a lesser extent to the trans-Golgi network, suggesting that cryptic peptides are representatives of this tumor cell compartment on the cell surface. Thus, we postulate that cryptic peptides are an important component of the immunological homunculus of the AT/RT cell (figure 7). However, in contrast to its fixed neurological counterpart, the immunological homunculus is most likely highly dynamic, since inflammation, ${ }^{63}$ but also therapeutic elements such as chemotherapy or kinase inhibitors can profoundly impact on HLA expression and unmask new peptide epitopes. ${ }^{64}$ Since the cryptic ligandome of only few tumors has been unveiled so far, we do not know whether the rather high contribution of cryptic peptides to the ligandome is an AT/RT-specific phenomenon and related to the epigenetic dysregulation of this tumor or whether frequencies of $>50 \%$ can be found in other tumors as well. Certainly, the stability and composition of the immunopeptidome during therapy and disease progression requires more active investigation.

A basic question in tumor vaccine design is whether selected peptides can give rise to $\mathrm{T}$ cell responses in vivo. We tried to address this issue on two levels: the level of de novo naïve $\mathrm{T}$ cell priming (mimicking a vaccine response) and on the level of existing recall responses in AT/RT patients. For level one testing, we chose a validated $\mathrm{T}$ cell assay in which highly purified naïve $\mathrm{CD} 8^{+} \mathrm{T}$ cells were primed on peptide-loaded autologous dendritic cells. ${ }^{33} 34$ In this assay, more than $80 \%$ of tested HLA-I peptides from natural, cryptic or neoantigenic origin yielded at least one positive response, suggesting that all three peptide sources are suitable for vaccine design. We found a weak, non-significant correlation between in silico 
HLA binding prediction and immunogenicity as well as a trend for more cytokine reactive $\mathrm{T}$ cells after stimulation with strong binding peptides. However, our analysis was not sufficiently powered to answer this question, so that the assumption that peptides with stronger HLA binding yield more IFN $\gamma$-producing $\mathrm{T}$ cells cannot be rejected. These data reflect a recent study on cytomegalovirus (CMV)-derived viral peptides in which also no clear correlation between HLA binding affinity and immunogenicity could be found. In this study, not all dominant CMV epitopes were contained within the $2 \%$ best binders and vice versa the group of strong binders harbored a high number of non-immunogenic peptides. ${ }^{65}$ Therefore, the association between in silico HLA binding rank and immunogenicity remains elusive. Peripheral lymphocytes of AT/RT patients showed no memory responses against the identified class-I peptides, indicating that patients are indeed 'CD8 naïve' for all three types of HLA class I peptides investigated here. In this context, it would have been interesting to test reactivity of AT/ RT tumor-infiltrating lymphocytes (TILs); however, this was precluded by scarcity of the available tumor material. Notably, in melanoma non-canonical peptides were not recognized by $\mathrm{CD} 8^{+}$TILs. ${ }^{61}$ It is conceivable that due to their exceptional short half-life cryptic peptides are not presented to $\mathrm{T}$ cells via dendritic cells in lymphatic tissue, that is, that the peripheral T-cell repertoire of AT/RT patients is devoid of these specificities, but these could be de novo primed by therapeutic vaccines. In contrast to missing responses against class I peptides, $\sim 83 \%$ of the tested HLA-II epitopes were recognized by patients' $\mathrm{T}_{\text {helper }}$ cells. This can partly be explained by the higher promiscuity of class II ligands for HLA-II binding and presentation. Yet, this reactivity was tumor specific since PBMCs of high-grade glioma patients recognized only $~ 50 \%$ of the tested HLA-II epitopes at a lower level, whereas CD4 $\mathrm{T}$ cells from healthy donors showed almost no reactivity. This indicates that the $\mathrm{T}_{\text {helper }}$ cell repertoire of AT/RT patients has been sensitized to tumor-associated class II epitopes during disease initiation. Furthermore, our data show a partial overlap of immunopeptidomes of two CNS tumors, GBM and AT/RTs on the level of natural and cryptic class I epitopes as well as by shared $\mathrm{CD} 4^{+}$recall responses. Eighteen per cent of the identified cryptic peptides were shared between AT/RTs and GBM, making these antigens highly promising candidates for future vaccines.

A limitation of our study certainly is that we worked predominantly with tumor banking material, which might have resulted in lower peptide yield due to suboptimal quality of material. The scarcity of tumor material in these small children made it impossible to determine the reactivity of AT/RT TILs against the different peptides. Furthermore, the lack of clinical information to these samples precluded an analysis between immunological findings and clinical outcome.

In summary, $\mathrm{AT} / \mathrm{RTs}$ as a paradigmatic example of a low-mutational pediatric cancer present a plethora of tumor-associated, immunogenic HLA class I and class II peptides. More than half of the class I peptides originated from non-canoncial translated proteins. Thus, personalized therapeutic vaccine cocktails should contain peptides from all three subgroups in order to have full representation of the entire tumor immunopeptidome in the vaccine. We could not identify entity-spanning HLA class I epitopes. However, our data show a broader overlap between different AT/RTs as well as with GBM samples in the cryptic class I and natural class II than in the natural class I ligandome. Therefore, in the quest for a broader applicable warehouse peptide pool, the former two ligandome compartments should be further studied more in depth, whereas natural class I peptides will remain the domain of individualized peptide cocktails.

\section{Author affiliations}

${ }^{1}$ Institute for Cell Biology, Department of Immunology, University of Tübingen,

Tubingen, Germany

${ }^{2}$ Rudolf-Virchow Zentrum, University Würzburg, Würzburg, Germany

${ }^{3}$ University Children's Hospital, University Medical Center Würzburg, Würzburg,

Germany

${ }^{4}$ Swabian Children's Cancer Center, Augsburg, Germany

${ }^{5}$ DKFZ Heidelberg, Heidelberg, Germany

${ }^{6}$ Department of Neuropathology, Institute for Pathology, University of Würzburg, Würzburg, Germany

${ }^{7}$ Cluster of Excellence iFIT (EXC2180), University of Tübingen, Tübingen, Germany

${ }^{8}$ Dr. Margarete Fischer-Bosch Institute of Clinical Pharmacology and Robert Bosch Center for Tumor Diseases (RBCT), Stuttgart, Germany

${ }^{9}$ Clinical Collaboration Unit Translational Immunology, German Cancer Consortium (DKTK), University Hospital of Tübingen, Tübingen, Germany

${ }^{10}$ Department of Neurosurgery, University Medical Center Würzburg, Würzburg,

Germany

${ }^{11}$ University Children's Hospital, University Medical Center Tübingen, Tübingen, Germany

${ }^{12}$ Department of Neurosurgery, University Medical Center Tübingen, Tübingen, Germany

${ }^{13}$ Department of Neurosurgery, Charité, Berlin, Germany

${ }^{14}$ Institute of Neuropathology, DGNN Brain Tumor Reference Center, University of Bonn, Bonn, Germany

${ }^{15}$ Institute for Transfusion Medicine, University Medical Center Würzburg, Würzburg, Germany

${ }^{16}$ University Children's Hospital, University Medical Center Hamburg-Eppendorf, Hamburg, Germany

${ }^{17}$ Department of Immunology, Weizmann Institute of Science, Rehovot, Israel

Acknowledgements We would like to thank Robert Wenzl for graphic design support with figure 7, Götz Gelbrich for aid with the statistical workup of IHC-data, and Stefan Stevanović for support with mass spectrometry data analysis and critical comments on the project progress.

Contributors Conceptualization: MEy, H-GR, and YR. Methodology: AM, H-GR, MW, and MEy. Investigation: AM, AS, NT, AK, PJ, JL, CMM, LMH, EK, MW, and FO. Provision of tumor material: JK, MS, UWT, MEb, MF, and TP. Visualization: AM and MEy. Writing - original draft: AM, AS, and MEy. Writing - review and editing: MEy, PGS, H-GR, and YR.

Funding EU-TRANSCAN JCT 2013 (ATRTPepVac), funding agency for Germany: Bundesministerium für Bildung und Forschung (BMBF), for Israel: Ministry of Health $(\mathrm{MoH})$ Parents Initiative Group for Children with Leukemia and Solid Tumors Würzburg e.V. Tour of Hope Foundation Elfrieda-Albert-Stiftung Vogel-Stiftung Dr. Eckernkamp.

Competing interests MEy has taken part in pediatric advisory boards of BMS and Atara Biotherapeutics and holds research collaborations with CellSource and Miltenyi Biotec.

Patient consent for publication Not required. 
Ethics approval Informed consent from the guardians of the patients was obtained following internal regulations of the institutional review board (Ethics Commission at the Faculty of Medicine, University of Würzburg, \#86/15).

Provenance and peer review Not commissioned; externally peer reviewed.

Data availability statement Data are available in a public, open access repository. The mass spectrometry proteomics data have been deposited to the ProteomeXchange Consortium (http://proteomecentral.proteomexchange.org) via the PRIDE partner repository (doi: 10.1093/nar/gky1106).

Supplemental material This content has been supplied by the author(s). It has not been vetted by BMJ Publishing Group Limited (BMJ) and may not have been peer-reviewed. Any opinions or recommendations discussed are solely those of the author(s) and are not endorsed by BMJ. BMJ disclaims all liability and responsibility arising from any reliance placed on the content. Where the content includes any translated material, BMJ does not warrant the accuracy and reliability of the translations (including but not limited to local regulations, clinical guidelines, terminology, drug names and drug dosages), and is not responsible for any error and/or omissions arising from translation and adaptation or otherwise.

Open access This is an open access article distributed in accordance with the Creative Commons Attribution Non Commercial (CC BY-NC 4.0) license, which permits others to distribute, remix, adapt, build upon this work non-commercially, and license their derivative works on different terms, provided the original work is properly cited, appropriate credit is given, any changes made indicated, and the use is non-commercial. See http://creativecommons.org/licenses/by-nc/4.0/.

\section{ORCID iD}

Matthias Eyrich http://orcid.org/0000-0002-8099-5903

\section{REFERENCES}

1 Frühwald MC, Hasselblatt M, Nemes K, et al. Age and DNA methylation subgroup as potential independent risk factors for treatment stratification in children with atypical teratoid/rhabdoid tumors. Neuro Oncol 2020;22:1006-17.

2 Versteege I, Sévenet N, Lange J, et al. Truncating mutations of hSNF5/INI1 in aggressive paediatric cancer. Nature 1998;394:203-6.

3 Johann PD, Erkek S, Zapatka M, et al. Atypical teratoid/rhabdoid tumors are comprised of three epigenetic subgroups with distinct enhancer landscapes. Cancer Cell 2016;29:379-93.

4 Torchia J, Picard D, Lafay-Cousin L, et al. Molecular subgroups of atypical teratoid rhabdoid tumours in children: an integrated genomic and clinicopathological analysis. Lancet Oncol 2015;16:569-82.

5 Vogelstein B, Papadopoulos N, Velculescu VE, et al. Cancer genome landscapes. Science 2013;339:1546-58.

6 Fumet J-D, Truntzer C, Yarchoan M, et al. Tumour mutational burden as a biomarker for immunotherapy: current data and emerging concepts. Eur J Cancer 2020;131:40-50.

$7 \mathrm{JQ}$ L, Wilson BA, Yong VW. Immune cell infiltrates in atypical teratoid/ rhabdoid tumors. Can J Neurol Sci 2012;39:605-12.

8 Melcher V, Graf M, Interlandi M, et al. Macrophage-tumor cell interaction promotes ATRT progression and chemoresistance. Acta Neuropathol 2020;139:913-36.

9 Chun H-JE, Johann PD, Milne K, et al. Identification and analyses of extra-cranial and cranial rhabdoid tumor molecular subgroups reveal tumors with cytotoxic T cell infiltration. Cell Rep 2019;29:2338-54.

10 Leruste A, Tosello J, Ramos RN, et al. Clonally expanded T cells reveal immunogenicity of rhabdoid tumors. Cancer Cell 2019;36:597-612.

11 Grabovska Y, Mackay A, O'Hare P, O'Hare P, et al. Pediatric pan-central nervous system tumor analysis of immune-cell infiltration identifies correlates of antitumor immunity. Nat Commun 2020;11:4324.

12 van Gool SW, Holm S, Rachor J, et al. Immunotherapy in atypical teratoid-rhabdoid tumors: data from a survey of the HGG-Immuno group. Cytotherapy 2016;18:1178-86.

13 Erhard F, Dölken L, Schilling B, et al. Identification of the cryptic HLA-I immunopeptidome. Cancer Immunol Res 2020;8:1018-26.

14 Laumont CM, Daouda T, Laverdure J-P, et al. Global proteogenomic analysis of human $\mathrm{MHC}$ class I-associated peptides derived from non-canonical reading frames. Nat Commun 2016;7:10238.

15 Bui H-H, Sidney J, Dinh K, et al. Predicting population coverage of T-cell epitope-based diagnostics and vaccines. BMC Bioinformatics 2006;7:153.

16 Scheel AH, Dietel M, Heukamp LC, et al. Harmonized PDL1 immunohistochemistry for pulmonary squamous-cell and adenocarcinomas. Mod Pathol 2016;29:1165-72.
17 Falk K, Rötzschke O, Stevanović S, et al. Allele-specific motifs revealed by sequencing of self-peptides eluted from $\mathrm{MHC}$ molecules. Nature 1991;351:290-6.

18 Kowalewski DJ, Schuster H, Backert L, et al. HLA ligandome analysis identifies the underlying specificities of spontaneous antileukemia immune responses in chronic lymphocytic leukemia (CLL). Proc Natl Acad Sci U S A 2015;112:E166-75.

19 Barnstable CJ, Bodmer WF, Brown G, et al. Production of monoclonal antibodies to group A erythrocytes, HLA and other human cell surface antigens-new tools for genetic analysis. Cell 1978;14:9-20.

20 Goldman JM, Hibbin J, Kearney L, et al. HLA-DA monoclonal antibodies inhibit the proliferation of normal and chronic granulocytic leukaemia myeloid progenitor cells. Br J Haematol 1982;52:411-20.

21 Pawelec G, Newman W, Schwulera U, et al. Heterogeneity of human natural killer recognition demonstrated by cloned effector cells and differential blocking of cytotoxicity with monoclonal antibodies. Cell Immunol 1985;92:31-40.

22 Boegel S, Löwer M, Bukur T, et al. HLA and proteasome expression body MAP. BMC Med Genomics 2018;11:36.

23 Käll L, Canterbury JD, Weston J, et al. Semi-supervised learning for peptide identification from shotgun proteomics datasets. Nat Methods 2007;4:923-5.

24 Lundegaard C, Lamberth K, Harndahl M, et al. NetMHC-3.0: accurate web accessible predictions of human, mouse and monkey MHC class I affinities for peptides of length 8-11. Nucleic Acids Res 2008;36:W509-12.

25 Jones DTW, Hutter B, Jäger N, et al. Recurrent somatic alterations of FGFR1 and ntrk2 in pilocytic astrocytoma. Nat Genet 2013;45:927-32

26 Rimmer A, Phan H, Mathieson I, et al. Integrating mapping-, assembly- and haplotype-based approaches for calling variants in clinical sequencing applications. Nat Genet 2014;46:912-8.

27 Doytchinova IA, Flower DR. VaxiJen: a server for prediction of protective antigens, tumour antigens and subunit vaccines. BMC Bioinformatics 2007;8:4.

28 Klatt MG, Kowalewski DJ, Schuster $\mathrm{H}$, et al. Carcinogenesis of renal cell carcinoma reflected in HLA ligands: a novel approach for synergistic peptide vaccination design. Oncoimmunology 2016;5:e1204504.

29 Calis JJA, Maybeno M, Greenbaum JA, et al. Properties of MHC class I presented peptides that enhance immunogenicity. PLoS Comput Biol 2013;9:e1003266.

30 Huang DW, Sherman BT, Lempicki RA. Bioinformatics enrichment tools: paths toward the comprehensive functional analysis of large gene Lists. Nucleic Acids Res 2009;37:1-13.

31 Szklarczyk D, Gable AL, Lyon D, et al. String v11: protein-protein association networks with increased coverage, supporting functional discovery in genome-wide experimental datasets. Nucleic Acids Res 2019;47:D607-13.

32 Hulsen T, de Vlieg J, Alkema W. BioVenn - a web application for the comparison and visualization of biological lists using areaproportional Venn diagrams. BMC Genomics 2008;9:488.

33 Wölfl M, Greenberg PD. Antigen-specific activation and cytokinefacilitated expansion of naive, human CD8+ T cells. Nat Protoc 2014;9:950-66.

34 Wölfl M, Merker K, Morbach $\mathrm{H}$, et al. Primed tumor-reactive multifunctional CD62L+ human CD8+ T cells for immunotherapy. Cancer Immunol Immunother 2011;60:173-86.

35 Kammula US, Lee KH, Riker Al. Functional analysis of antigenspecific T lymphocytes by serial measurement of gene expression in peripheral blood mononuclear cells and tumor specimens. J Immunol 1999;163:6867-75.

36 Zhang J, Xin L, Shan B, et al. Peaks DB: de novo sequencing assisted database search for sensitive and accurate peptide identification. Mol Cell Proteomics 2012;11:M111.010587.

37 Shraibman B, Barnea E, Kadosh DM, et al. Identification of tumor antigens among the HLA peptidomes of glioblastoma tumors and plasma. Mol Cell Proteomics 2018;17:2132-45.

38 Vita R, Overton JA, Greenbaum JA, et al. The immune epitope database (IEDB) 3.0. Nucleic Acids Res 2015;43:D405-12.

39 Marcu A, Bichmann L, Kuchenbecker L, et al. HLA ligand atlas: a benign reference of HLA-presented peptides to improve T-cell-based cancer immunotherapy. J Immunother Cancer 2021;9:e002071.

40 Dutoit V, Herold-Mende C, Hilf N, et al. Exploiting the glioblastoma peptidome to discover novel tumour-associated antigens for immunotherapy. Brain 2012;135:1042-54.

41 Peper JK, Bösmüller H-C, Schuster H, et al. HLA ligandomics identifies histone deacetylase 1 as target for ovarian cancer immunotherapy. Oncoimmunology 2016;5:e1065369. 
42 Engels B, Engelhard VH, Sidney J, et al. Relapse or eradication of cancer is predicted by peptide-major histocompatibility complex affinity. Cancer Cell 2013;23:516-26.

43 Aleksic M, Liddy N, Molloy PE, et al. Different affinity windows for virus and cancer-specific T-cell receptors: implications for therapeutic strategies. Eur J Immunol 2012;42:3174-9.

44 Ott PA, Hu Z, Keskin DB, et al. An immunogenic personal neoantigen vaccine for patients with melanoma. Nature 2017;547:217-21.

45 Bassani-Sternberg M, Bräunlein E, Klar R, et al. Direct identification of clinically relevant neoepitopes presented on native human melanoma tissue by mass spectrometry. Nat Commun 2016;7:13404.

46 Newey A, Griffiths B, Michaux J, et al. Immunopeptidomics of colorectal cancer organoids reveals a sparse HLA class I neoantigen landscape and no increase in neoantigens with interferon or MEK-inhibitor treatment. J Immunother Cancer 2019;7:309.

47 Purbhoo MA, Irvine DJ, Huppa JB, et al. T cell killing does not require the formation of a stable mature immunological synapse. Nat Immunol 2004;5:524-30.

48 Marijt KA, Doorduijn EM, van Hall T. TEIPP antigens for T-cell based immunotherapy of immune-edited HLA class Ilow cancers. Mol Immunol 2019;113:43-9.

49 Laumont CM, Vincent K, Hesnard L, et al. Noncoding regions are the main source of targetable tumor-specific antigens. Sci Trans/ Med 2018;10:eaau5516.

50 Zhao Q, Laverdure J-P, Lanoix J, et al. Proteogenomics uncovers a vast repertoire of shared tumor-specific antigens in ovarian cancer. Cancer Immunol Res 2020;8:544-55.

51 Lee RS, Stewart C, Carter SL, et al. A remarkably simple genome underlies highly malignant pediatric rhabdoid cancers. J Clin Invest 2012;122:2983-8.

52 VIková V, Štěpánek I, Hrušková V, et al. Epigenetic regulations in the IFN $\gamma$ signalling pathway: IFN $\gamma$-mediated MHC class I upregulation on tumour cells is associated with DNA demethylation of antigenpresenting machinery genes. Oncotarget 2014;5:6923-35.

53 Garrido F, Aptsiauri N, Doorduijn EM, et al. The urgent need to recover MHC class I in cancers for effective immunotherapy. Curr Opin Immunol 2016;39:44-51.
54 Löffler MW, Kowalewski DJ, Backert L, et al. Mapping the HLA Ligandome of colorectal cancer reveals an imprint of malignant cell transformation. Cancer Res 2018;78:4627-41.

55 Neumann A, Hörzer H, Hillen N, et al. Identification of HLA ligands and T-cell epitopes for immunotherapy of lung cancer. Cancer Immunol Immunother 2013;62:1485-97.

56 Stickel JS, Stickel N, Hennenlotter J, et al. Quantification of HLA class I molecules on renal cell carcinoma using Edman degradation. BMC Urol 2011;11:1.

57 Bilich T, Nelde A, Bauer J, et al. Mass spectrometry-based identification of a B-cell maturation antigen-derived T-cell epitope for antigen-specific immunotherapy of multiple myeloma. Blood Cancer J 2020;10.

58 Backert L, Kowalewski DJ, Walz S, et al. A meta-analysis of HLA peptidome composition in different hematological entities: entityspecific dividing lines and "pan-leukemia" antigens. Oncotarget 2017;8:43915-24.

59 Chen B, Khodadoust MS, Olsson N, et al. Predicting HLA class II antigen presentation through integrated deep learning. Nat Biotechnol 2019;37:1332-43.

60 Ruiz Cuevas MV, Hardy M-P, Hollý J, et al. Most non-canonical proteins uniquely populate the proteome or immunopeptidome. Cell Rep 2021;34:108815.

61 Chong C, Müller M, Pak H, et al. Integrated proteogenomic deep sequencing and analytics accurately identify non-canonical peptides in tumor immunopeptidomes. Nat Commun 2020;11:1293.

62 Neidert MC, Kowalewski DJ, Silginer M, et al. The natural HLA ligandome of glioblastoma stem-like cells: antigen discovery for $\mathrm{T}$ cell-based immunotherapy. Acta Neuropathol 2018;135:923-38.

63 Murata S, Takahama Y, Kasahara M, et al. The immunoproteasome and thymoproteasome: functions, evolution and human disease. Nat Immunol 2018;19:923-31.

64 Oh CY, Klatt MG, Bourne C, et al. Alk and RET inhibitors promote HLA class I antigen presentation and unmask new antigens within the tumor immunopeptidome. Cancer Immunol Res 2019;7:1984-97.

65 Lübke M, Spalt S, Kowalewski DJ, et al. Identification of HCMVderived T cell epitopes in seropositive individuals through viral deletion models. J Exp Med 2020;217. doi:10.1084/jem.20191164. [Epub ahead of print: 0203 2020]. 